\title{
Plasticity with generalized hardening: constitutive modeling and computational aspects
}

\author{
Claudio Tamagnini · Matteo O. Ciantia
}

\begin{abstract}
In this work, an extended theory of plasticity with generalized hardening is proposed to describe the response of geomaterials under both mechanical and environmental processes, which include as special cases several elastoplastic constitutive equations proposed in the literature to model such processes as 10 desaturation or suction hardening, thermal softening, chemo-mechanical coupling effects in fine-grained soils, as well as weathering of soft rocks. In the formulation of the theory, the coupling between mechanical and environmental processes takes place at two levels: first, as an additional direct contribution to the constitutive stress changes, taking place in both elastic and elastoplastic processes; and, second, as a result of the evolution of the internal state variables induced by changes in the environmental process variables. This last effect is incorporated through a set of generalized hardening rules. As an example of application, the general formulation is specialized to the particular case of weak calcarenite rocks undergoing degradation processes due to plastic deformations, changes in degree of saturation (short-term debonding) and chemical dissolution of the bond material and the solid grains (long-term debonding). The resulting model is implemented in a FE code by means of an implicit Generalized Backward Euler algorithm, suitably modified to incorporate the full formalism of plasticity with generalized hardening. Results of numerical simulations carried out at the element level show the accuracy and 25 efficiency properties of the proposed stress-point algorithm. The simulation of a representative initial-boundary value problem demonstrate the practical relevance of environmental degradation effects in practical applications, over periods of time comparable with the life cycle of most geotechnical structures.
\end{abstract}

Keywords Plasticity with generalized hardening · Environmental loading • 30 Coupled multiphysics processes - Computational plasticity

Claudio Tamagnini

University of Perugia, Perugia (Italy)

Matteo Oryem Ciantia

Imperial College of Science, Technology and Medicine, London (UK) 


\section{Introduction}

In his recent Rankine Lecture [20], Antonio Gens pointed out that a remarkable number of recent developments in the field of constitutive modeling of geomaterials have been focusing on the modification of classical approaches to describe the

35 effects of the interactions between the soil (or rock) mass and the environment - intended in a very broad sense to include all the possible coupled phenomena involving changes in pore fluid pressures and degree of saturation (hydraulic coupling), temperature (thermal coupling) and chemical composition of the solid and liquid phases (chemical interaction) - and on the development of appropriate 40 constitutive theories capable of quantitatively reproducing the observed behavior under both mechanical and environmental loading conditions.

In particular, a number of modifications to the classical theory of hardening elastoplasticity have been proposed in the context of geomechanics to describe a number of practically relevant aspects of the mechanical behavior of geomateri4 als. A common, distinctive feature of those constitutive theories is that the size and shape of the yield locus, as well as its evolution with the loading process are assumed to depend to accumulated plastic strains and on the evolution of other environmental process variables, such as degree of saturation (or suction), temperature, concentrations of chemical species in the liquid phase. In particular, we 50 recall:

i) elastoplastic models for unsaturated soil (formulated in terms of average skeleton stress and suction) in which an explicit dependence of the size of the yield surface on the degree of saturation or suction (desaturation or suction hardening) is assumed to reproduce the phenomenon of collapse upon wetting in unsaturated soils (see, e.g., [24,43,16]);

ii) thermoplastic models for rocks [29] and soils $[21,7,32,22,44]$, in which the internal variables depend on the temperature $T$ (thermal softening);

iii) extensions of classical elastoplasticity advocated by [30], [42], [31] to model chemical degradation effects in cemented granular soils or weak rocks, in which some bonding-related internal variables are subject to both mechanical and chemical degradation, or chemoplastic models proposed by [23], [27] to describe chemo-mechanical coupling effects in fine-grained soils.

It is worth noting that, in most cases, the effect of the changes in environmental variables on the elastic domain of the material is introduced by extending the 5 hardening laws of the material to allow the internal variables to change with the degree of saturation (or suction), temperature, or concentration of chemical species in the pore fluid, other than with the accumulated plastic strains.

The first goal of this paper is to show that a general theory of plasticity with generalized hardening - which includes as a special case most of the aforemen70 tioned works - can be constructed on the assumption that the coupling between mechanical and environmental processes takes place at two levels:

a) as an additional direct contribution to the constitutive stress changes (e.g., suction-induced stress relaxation; thermal expansion/contraction effects, chemical damage effects) taking place even for purely elastic processes; and,

b) as a consequence of the evolution of the internal state variables of the material with the changes in the environmental process variables. 
This last effect is incorporated through a set of generalized hardening rules. The fundamental assumptions behind the development of the theory of plasticity with generalized hardening and the development of the constitutive equations in rateorm are provided in Sect. 2.

In Sect. 3 it is shown how the general formulation can be specialized to particular cases, focusing on the constitutive model for unsaturated, soft porous rocks such as calcarenites, chalks and porous sandstones, recently presented by Ciantia and di Prisco [10]. The mechanical behavior of calcarenite rocks has been inten85 sively investigated in refs. [11-13,9]. From a detailed microstructural investigation of Gravina calcarenite, Ciantia and Hueckel [12] and Ciantia et al. [8] have identified two distinct types of intergranular bonds, characterized by different strengths and formation mechanisms: strong diagenetic bonds, made of bridges of calcite formed through a diagenetic process, and weak depositional bonds, formed by a 90 mixture of the calcite "powder" and seawater salts that precipitates as sediment into a meniscus-shaped bond when water evaporates. The degradation processes affecting these bonds are of two different kinds:

a) short-term debonding (STD), associated to the increase of the degree of saturation of the material, due to the rapid destruction of depositional bonds as the micrograins of calcite returns in suspension in the pore water;

b) long-term debonding (LTD), due to the chemical dissolution of the diagenetic bonds of calcite, taking place when the rock is flooded by water for a long period of time, under fully saturated conditions.

The chemical reactions between the species dissolved in the pore liquid and the individual grains of the solid skeleton can also be responsible for the occurrence of significant grain dissolution, which, in turn, can be responsible for chemicallyinduced volumetric deformations of the solid skeleton.

The three degradation processes mentioned are characterized by progressively increasing time scales. In this respect, it is worth noting that even the slowest degradation processes may produce significant effects over time periods comparable to the life cycle of most geotechnical engineering structures, and are thus of significant practical relevance. In fact, the progressive loss of strength caused by the different kind of degradation processes has potentially dangerous consequences for the anthropic environment, associated to slope stability problems or collapse of underground cavities, see e.g.[34].

The application of the constitutive models developed within this theoretical framework to practical engineering problems requires the development of accurate and robust numerical procedures to integrate (at the Gauss point level, in a typical displacement-based FE implementation) the constitutive equations in rate form along the prescribed loading history. The second main goal of the paper is to present a robust and accurate numerical implementation strategy for isotropic plasticity with generalized hardening - based on a straightforward modification of the implicit Backward-Euler stress-point algorithm of Tamagnini et al. [42] - and its application to the Ciantia and di Prisco model. The integration algorithm and 120 its linearization, to be used in the determination of the Jacobian matrices employed in the iterative solution of the discrete equilibrium problem via Newton's method, are described in Sects. 4 and 5.

A few numerical simulations of element tests and initial-boundary value problems are presented in Sect. 6 in order to demonstrate the the accuracy and effi- 
ciency properties of the proposed stress-point algorithm. Finally some concluding remarks are provided in Sect. 7 .

\section{Fundamental assumptions and constitutive equations in rate-form}

In order to keep the theory as simple as possible, in the following the material response will be assumed to be isotropic, and the derivations will be restricted to

the case of linearized kinematics ( "small strains" assumption).

2.1 Kinematic decomposition, environmental process variables and constitutive stresses

As customary for small strain kinematics, the total strain rate $\dot{\boldsymbol{\epsilon}}$ is assumed to be additively decomposed into an elastic part, $\dot{\boldsymbol{\epsilon}}^{e}$, and a plastic, irreversible part, $\dot{\boldsymbol{\epsilon}}^{p}$ :

$$
\dot{\boldsymbol{\epsilon}}=\dot{\boldsymbol{\epsilon}}^{e}+\dot{\boldsymbol{\epsilon}}^{p}
$$
a suitable environmental process variables vector $\boldsymbol{\alpha} \in \mathbb{R}^{m}$, defined as:

$$
\boldsymbol{\alpha}=\left\{S_{r}, T, c_{1}, c_{2}, \ldots\right\}^{T}
$$

In eq. (2), $S_{r}$ is the degree of saturation (equal to 1 for saturated states), $T$ is the temperature, and $c_{k}$ are the concentrations of $n_{c}$ chemical species present in the solid skeleton or dissolved in the pore liquid which may be responsible for chemo/mechanical processes, such as dissolution, precipitation, cation exchange, etc.

In a unsaturated state, the description of the mechanical response of the material requires the use of two independent constitutive stress quantities. As observed by [20], there are several possible alternative choices for the first constitutive stress

a) the net stress tensor

$$
\bar{\sigma}=\tau-u_{g} \mathbf{1}
$$

where $\boldsymbol{\tau}$ is the total stress tensor, $u_{g}$ the pore gas pressure and $\mathbf{1}$ the second order identity tensor;

b) the average skeleton stress

$$
\boldsymbol{\sigma}^{\prime \prime}=\boldsymbol{\tau}-\left\{\left(1-S_{r}\right) u_{g}+S_{r} u_{\ell}\right\} \mathbf{1}
$$

where $u_{\ell}$ is the pore liquid pressure.

The first choice has been adopted, e.g., by [1], [45], [15]; the second constitutive stress has been introduced by Schrefler [37] and has been used, e.g., by [25], [2], [24], [38], [4], [16].

In the following, to keep the description of the theory sufficiently general, we will denote with the symbol $\boldsymbol{\sigma}$ the stress quantity adopted as the first constitutive variable, and use the suction $s=u_{g}-u_{\ell}$ as the second constitutive variable. For to the classical Terzaghi effective stress. 


\subsection{Elastic behavior}

The elastic response of the material is defined by postulating a (hypo)elastic relation between $\dot{\boldsymbol{\sigma}}$ and $\dot{\boldsymbol{\epsilon}}^{e}$ of the form:

$$
\dot{\boldsymbol{\sigma}}=\boldsymbol{D}^{e}\left(\dot{\boldsymbol{\epsilon}}-\dot{\boldsymbol{\epsilon}}^{p}\right)+\boldsymbol{M} \dot{\boldsymbol{\alpha}}
$$

where $\boldsymbol{D}^{e}$ is the elastic tangent stiffness tensor, and

$$
\boldsymbol{M}=\left[\boldsymbol{M}_{S_{r}}, \boldsymbol{M}_{T}, \boldsymbol{M}_{c_{1}}, \boldsymbol{M}_{c_{2}}, \ldots\right]
$$

is a coupling matrix accounting for such effects as changes in suction, thermal expansion/contraction and deformations induced by modifications in the chemical composition of the pore liquid or by grain dissolution.

For example, in a linear elastic, isotropic soil subject to heating we would have:

$$
M_{T}=\beta K 1
$$

where $K$ is the bulk modulus of the solid skeleton, $\beta$ is the thermal expansion coefficient and $\mathbf{1}$ is the second order identity tensor.

\subsection{Elastic domain, yield surface and flow rule}

Irreversibility of the material response is introduced by requiring that the state of the material, defined in terms of the stress tensor $\boldsymbol{\sigma}$ and the internal variable pseudo-vector $\boldsymbol{q} \in \mathbb{R}^{n}$, to lie in the convex set:

$$
\mathbb{E}_{\sigma}:=\{(\boldsymbol{\sigma}, \boldsymbol{q}) \mid f(\boldsymbol{\sigma}, \boldsymbol{q}) \leq 0\}
$$

where $f: \operatorname{Sym} \times \mathbb{R}^{n} \mapsto \mathbb{R}$ is the yield function of the material.

According to eq. (5), the actual shape and size of the yield locus may be affected by the previous loading history as well as by the evolution of the nonmechanical process variables through the internal variables $\boldsymbol{q}$. Note that, due to the assumption of isotropy, all the internal variables collected in vector $\boldsymbol{q}$ are scalars.

For many natural geomaterials, such as bonded granular soils or weak rocks, it is useful to consider $\boldsymbol{q}$ as composed of two parts:

$$
\boldsymbol{q}=\left\{\boldsymbol{q}_{u}^{T}, \boldsymbol{q}_{b}^{T}\right\}^{T}
$$

The vector $\boldsymbol{q}_{u}$ collects the internal variables quantifying history dependence of the reference unbonded material, while the vector $\boldsymbol{q}_{b}$ contains the bonding-related internal variables. As detailed in the following Sect. 2.4, both quantities can be affected by plastic deformations as well as changes in the environmental process variables.

The plastic strain rate tensor is provided by a standard flow rule:

$$
\dot{\boldsymbol{\epsilon}}^{p}=\dot{\gamma} \boldsymbol{Q}(\boldsymbol{\sigma}, \boldsymbol{q}) \quad \boldsymbol{Q}:=\frac{\partial g}{\partial \boldsymbol{\sigma}}(\boldsymbol{\sigma}, \boldsymbol{q})
$$

where $g:$ Sym $\times \mathbb{R}^{n} \mapsto \mathbb{R}$ is the plastic potential function, and $\dot{\gamma}$ is the plastic multiplier, subject to the standard Kuhn-Tucker complementarity conditions:

$$
f(\boldsymbol{\sigma}, \boldsymbol{q}) \leq 0 \quad \dot{\gamma} \geq 0 \quad \dot{\gamma} f(\boldsymbol{\sigma}, \boldsymbol{q})=0
$$

According to eq. (8), plastic processes, characterized by $\dot{\gamma}>0$, may occur only for states on the yield surface, where $f=0$. 
2.4 Generalized hardening laws

The evolution of the internal variables is provided by the following generalized hardening law:

$$
\dot{\boldsymbol{q}}=\dot{\gamma} \boldsymbol{h}(\boldsymbol{\sigma}, \boldsymbol{q}, \boldsymbol{\alpha})+\boldsymbol{N}(\boldsymbol{\sigma}, \boldsymbol{q}, \boldsymbol{\alpha}) \dot{\boldsymbol{\alpha}}
$$

where $\boldsymbol{h} \in \mathbb{R}^{n}$ and

$$
\boldsymbol{N}=\left[\boldsymbol{N}_{S_{r}}, \boldsymbol{N}_{T}, \boldsymbol{N}_{c_{1}}, \boldsymbol{N}_{c_{2}}, \ldots\right]
$$

are suitable hardening functions. The first term on the RHS of eq. (9) describes the changes in the internal variables associated with plastic deformations, while the second term accounts for all non-mechanical hardening/softening processes induced by a change of environmental process variables $\boldsymbol{\alpha}$.

This formulation of the evolution equations for the internal variables incorporate, as special cases, a number of well-known experimentally observed features of the hardening/softening processes experienced by natural geomaterials upon environmental loading, such as desaturation hardening, thermal softening, chemo-mechanical coupling in fine-grained soils and chemical degradation effects in bonded granular materials or soft rocks.

\subsection{Consistency condition and constitutive equations in rate-form}

The consistency condition $(\dot{\gamma} \dot{f}=0)$ applied to a plastic loading process allows to determine the plastic multiplier as a function of the rates of the process variables. Let:

$$
\boldsymbol{P}:=\frac{\partial f}{\partial \boldsymbol{\sigma}} \quad \text { and } \quad \boldsymbol{W}:=\frac{\partial f}{\partial \boldsymbol{q}}
$$

denote the gradients of the yield $f$ with respect to $\boldsymbol{\sigma}$ and $\boldsymbol{q}$. By exploiting the elastic constitutive equation (3) and the generalized flow rule (9), the following expression for the plastic multiplier is obtained:

$$
\dot{\gamma}=\frac{1}{K_{p}}\left\langle\boldsymbol{P} \cdot \boldsymbol{D}^{e} \dot{\boldsymbol{\epsilon}}+\boldsymbol{P} \cdot \boldsymbol{M} \dot{\boldsymbol{\alpha}}+\boldsymbol{W} \cdot \boldsymbol{N} \dot{\boldsymbol{\alpha}}\right\rangle
$$

where $\langle x\rangle:=(x+|x|) / 2$ denotes the ramp function, and:

$$
K_{p}:=\boldsymbol{P} \cdot \boldsymbol{D}^{e} \boldsymbol{Q}-\boldsymbol{W} \cdot \boldsymbol{h}=\boldsymbol{P} \cdot \boldsymbol{D}^{e} \boldsymbol{Q}+H_{p}
$$

the scalar $H_{p}=-\boldsymbol{W} \cdot \boldsymbol{h}$ being the hardening modulus of the material.

The derivation of eq. (11) requires that $K_{p}$ is strictly positive. For elastoplastic materials without chemical degradation it corresponds to the usual exclusion of subcritical softening, as first defined by [28]. As discussed, e.g., by [40], this last assumption is crucial in the establishment of the correct formulation of the loading/unloading conditions in presence of softening. Recently, [14] have shown that this limitation of the classical theory can be circumvented, through an adaptive viscoplastic regularization for the equations of the rate-independent evolution problem.

It is worth noting that eq. (10) can be interpreted as follows:

$$
\dot{\gamma}=\dot{\gamma}_{\text {mec }}+\dot{\gamma}_{\text {env }} \geq 0
$$


with:

$$
\begin{aligned}
\dot{\gamma}_{\mathrm{mec}} & :=\frac{1}{K_{p}} \boldsymbol{P} \cdot \boldsymbol{D}^{e} \dot{\boldsymbol{\epsilon}}=\frac{1}{K_{p}} \boldsymbol{P} \cdot(\dot{\boldsymbol{\sigma}})_{\boldsymbol{\alpha}=\mathrm{const}}^{\mathrm{tr}} \\
\dot{\gamma}_{\mathrm{env}} & :=\frac{1}{K_{p}}(\boldsymbol{P} \cdot \boldsymbol{M} \dot{\boldsymbol{\alpha}}+\boldsymbol{W} \cdot \boldsymbol{N} \dot{\boldsymbol{\alpha}})
\end{aligned}
$$

While $\dot{\gamma}_{\text {mec }}$ coincides with the plastic multiplier of classical elastoplasticity, see, e.g., [40], $\dot{\gamma}_{\text {env }}$ accounts for the effect of environmental processes. These affect the response of the material in two different ways: by contributing directly to the evolution of the constitutive stress through the coupling term $\boldsymbol{M} \dot{\boldsymbol{\alpha}}$ in eq. (3), and by contributing to the evolution of the internal variables through the term $\boldsymbol{N} \dot{\boldsymbol{\alpha}}$ in eq. (9).

For plastic loading to occur, the sum of $\dot{\gamma}_{\text {mec }}$ and $\dot{\gamma}_{\text {env }}$ needs to be positive, while one of the two terms may still be negative. In particular, plastic strains may occur even if the mechanical trial stress rate $(\dot{\boldsymbol{\sigma}})_{\boldsymbol{\alpha}=\text { const. is pointing inwards }}^{\mathrm{tr}}$ the current yield locus $\left(\dot{\gamma}_{\text {mec }}<0\right)$, provided that the changes in environmental variables $\boldsymbol{\alpha}$ determine a reduction in size of the elastic domain sufficiently large to keep the plastic multiplier positive, as it occurs, for example, in the case of chemical degradation [42].

Substituting the expression (11) for the plastic multiplier in the evolution equations (3) - where the flow rule (7) is used to express the plastic strain rate - and (9), we obtain, after some algebra:

$$
\begin{gathered}
\dot{\boldsymbol{\sigma}}=\boldsymbol{D}^{e p} \dot{\boldsymbol{\epsilon}}+\boldsymbol{M}^{e p} \dot{\boldsymbol{\alpha}} \\
\dot{\boldsymbol{q}}=\boldsymbol{H}^{p} \dot{\boldsymbol{\epsilon}}+\boldsymbol{N}^{e p} \dot{\boldsymbol{\alpha}}
\end{gathered}
$$

where:

$$
\begin{gathered}
\boldsymbol{D}^{e p}:=\boldsymbol{D}^{e}-\frac{\mathcal{H}(\dot{\gamma})}{K_{p}}\left(\boldsymbol{D}^{e} \boldsymbol{Q}\right) \otimes\left(\boldsymbol{P}^{T} \boldsymbol{D}^{e}\right) \\
\boldsymbol{M}^{e p}:=\boldsymbol{M}-\frac{\mathcal{H}(\dot{\gamma})}{K_{p}}\left(\boldsymbol{D}^{e} \boldsymbol{Q}\right) \otimes\left(\boldsymbol{P}^{T} \boldsymbol{M}+\boldsymbol{W}^{T} \boldsymbol{N}\right) \\
\boldsymbol{H}^{p}:=\frac{\mathcal{H}(\dot{\gamma})}{K_{p}} \boldsymbol{h} \otimes\left(\boldsymbol{P}^{T} \boldsymbol{D}^{e}\right) \\
\boldsymbol{N}^{e p}:=\boldsymbol{N}+\frac{\mathcal{H}(\dot{\gamma})}{K_{p}} \boldsymbol{h} \otimes\left(\boldsymbol{P}^{T} \boldsymbol{M}+\boldsymbol{W}^{T} \boldsymbol{N}\right)
\end{gathered}
$$

and $\mathcal{H}(\dot{\gamma})$ is the Heaviside step function, equal to one if $\dot{\gamma}>0$ and zero otherwise.

Finally, the formulation is completed by providing the evolution equation for the second constitutive variable, namely the suction $s$ :

$$
\dot{s}=\boldsymbol{k}_{\epsilon}(\boldsymbol{\sigma}, \boldsymbol{q}, \boldsymbol{\alpha}) \cdot \dot{\boldsymbol{\epsilon}}+\boldsymbol{k}_{\alpha}(\boldsymbol{\sigma}, \boldsymbol{q}, \boldsymbol{\alpha}) \cdot \dot{\boldsymbol{\alpha}}
$$

where $\boldsymbol{k}_{\epsilon}$ and $\boldsymbol{k}_{\alpha}$ are suitable constitutive functions which generalize the concept of soil-water retention curve used in unsaturated soil modeling to more complex environmental loading conditions. 


\section{Application to environmental degradation of calcarenite rocks}

As an example of application of the general theory to a specific class of geomaterials of relevant practical interest, we consider in this section the constitutive model recently proposed by Ciantia and di Prisco [10] for unsaturated, soft porous rocks such as calcarenites, chalks and porous sandstones, quite common in the Mediterranean region. In this section, the main constitutive functions adopted in the model are briefly recalled to outline their relation with the general theory.

\subsection{Environmental process variables and constitutive stresses}

Under isothermal conditions, considering that the STD process is associated to

the progressive increase of the degree of saturation of the solid skeleton, and that LTD and GDP processes are the consequence of a progressive loss of mass in the solid skeleton caused by chemical dissolutions, the environmental process variables vector $\boldsymbol{\alpha}$ of eq. (2) reduces to:

$$
\boldsymbol{\alpha}=\left\{S_{r}, \xi_{d}\right\}^{T}
$$

where $\xi_{d} \in[0,1)$, defined as:

$$
\xi_{d}(t)=-\frac{\Delta m_{s}(t)}{m_{s 0}}=\frac{m_{s 0}-m_{s}(t)}{m_{s 0}}
$$

is the mass dissolution ratio, quantifying the relative amount of mass loss due to bonds and grains dissolution.

In the model formulation, the average skeleton stress $\sigma^{\prime \prime}$ (in the following simply denoted by $\boldsymbol{\sigma}$ ) and the suction $s$ are adopted as constitutive stresses. In view of the large dimensions of the macropores and of the high stiffness of the solid skeleton, the soil-water characteristic curve is assumed to be given by a simple one-to-one function $S_{r}=S_{r}(s)$, neglecting any coupling effect with the mechanical deformation of the solid skeleton and the chemical dissolution process.

\subsection{Elastic behavior}

As in Tamagnini et al. [42], the (hyper)elastic response of the material is defined by prescribing a suitable stored energy function $\psi$, which, in this case, incorporates an isotropic damage variable $\mathcal{D}$ to take into account the effects of environmental debonding on the elastic properties of the material:

$$
\psi\left(\boldsymbol{\epsilon}^{e}, \boldsymbol{\alpha}\right)=\psi_{0}\left(\boldsymbol{\epsilon}^{e}\right)\left\{1+\delta_{b}(1-\mathcal{D})\right\}
$$

In eq. (25), $\psi_{0}\left(\boldsymbol{\epsilon}^{e}\right)$ represents the stored energy function of the fully unbonded granular soil, while $\delta_{b}$ is a material constants. The damage variable $\mathcal{D} \in[0,1]$ accounts for the effects of STD and LTD processes on material stiffness, and, as such, it depends on the environmental process variables $S_{r}$ and $\xi_{d}$. A similar approach has been used in [35] and [36] to model chemical degradation of concrete. The definition of the function $\mathcal{D}\left(S_{r}, \xi_{d}\right)$ is provided in the following Sect. 3.5. 
By taking the first and second derivatives of eq. (25) with respect to $\epsilon^{e}$, the following expressions for the constitutive stress tensor, the elastic tangent stiffness $\boldsymbol{D}^{e}$ and the elastic-damage coupling matrix $\boldsymbol{M}_{d}$ are obtained:

$$
\begin{gathered}
\boldsymbol{\sigma}=\left\{1+\delta_{b}(1-\mathcal{D})\right\} \frac{\partial \psi_{0}}{\partial \boldsymbol{\epsilon}^{e}} \\
\boldsymbol{D}^{e}:=\left\{1+\delta_{b}(1-\mathcal{D})\right\} \frac{\partial^{2} \psi_{0}}{\partial \boldsymbol{\epsilon}^{e} \otimes \partial \boldsymbol{\epsilon}^{e}} \\
\boldsymbol{M}_{d}=-\delta_{b} \frac{\partial \psi_{0}}{\partial \boldsymbol{\epsilon}^{e}} \otimes \frac{\partial \mathcal{D}}{\partial \boldsymbol{\alpha}}
\end{gathered}
$$

In Ciantia and di Prisco model, the function $\psi_{0}$ is taken from refs. [5,42]. This allows to model a linear dependence of the apparent bulk and shear stiffness coefficients on mean stress $p=\operatorname{tr}(\boldsymbol{\sigma}) / 3$, as well as the occurrence of volumetricdeviatoric coupling for non-isotropic stress states.

\subsection{Yield surface and plastic potential}

Due to the assumption of material isotropy, the internal state variables collected in vector $\boldsymbol{q}$ are all scalars and the yield function $f$ and the plastic potential $g$ depend on the average skeleton stress $\boldsymbol{\sigma}$ through its invariants $(p, q, \theta)$.

As in [42] and [31], the functions proposed by [26] have been adopted in the model formulation, for their flexibility in reproducing a wide range of experimental observations:

$$
\begin{aligned}
f\left(p, q, \theta, p_{s}, p_{m}\right) & =A_{f}^{K_{1 f} / C_{f}} B_{f}^{-K_{2 f} / C_{f}} p^{*}-p_{c}^{*}=0 \\
g\left(p, q, \theta, p_{m}\right) & =A_{g}^{K_{1 g} / C_{g}} B_{g}^{-K_{2 g} / C_{g}} p^{*}-\tilde{p}_{c}^{*}=0
\end{aligned}
$$

where:

$$
\begin{aligned}
K_{1 \alpha}:=\frac{m_{\alpha}\left(1-a_{\alpha}\right)}{2\left(1-m_{\alpha}\right)}\left\{1+\sqrt{1-\frac{4 a_{\alpha}\left(1-m_{\alpha}\right)}{m_{\alpha}\left(1-a_{\alpha}\right)^{2}}}\right\} \\
K_{2 \alpha}:=\frac{m_{\alpha}\left(1-a_{\alpha}\right)}{2\left(1-m_{\alpha}\right)}\left\{1-\sqrt{1-\frac{4 a_{\alpha}\left(1-m_{\alpha}\right)}{m_{\alpha}\left(1-a_{\alpha}\right)^{2}}}\right\} \\
A_{\alpha}:=1+\frac{1}{K_{1 \alpha} M_{\alpha}} \frac{q}{p^{*}} \\
B_{\alpha}:=1+\frac{1}{K_{2 \alpha} M_{\alpha}} \frac{q}{p^{*}} \\
C_{\alpha}:=\left(1-m_{\alpha}\right)\left(K_{1 \alpha}-K_{2 \alpha}\right)
\end{aligned}
$$

with $\alpha=f$ or $g$, and:

$$
p^{*}:=p+p_{t} \quad p_{c}^{*}:=p_{s}+(1+k) p_{t} \quad \tilde{p}_{c}^{*}:=\tilde{p}_{c}+p_{t}
$$

In the above expressions, $\left\{p_{s}\right\} \equiv \boldsymbol{q}_{u}$ and $\left\{p_{t}\right\} \equiv \boldsymbol{q}_{b}$ are the hardening variables describing the effects of previous (mechanical and non-mechanical) loading history; $\tilde{p}_{c}$ is a dummy parameter to be determined from the current state setting $g=0$, 


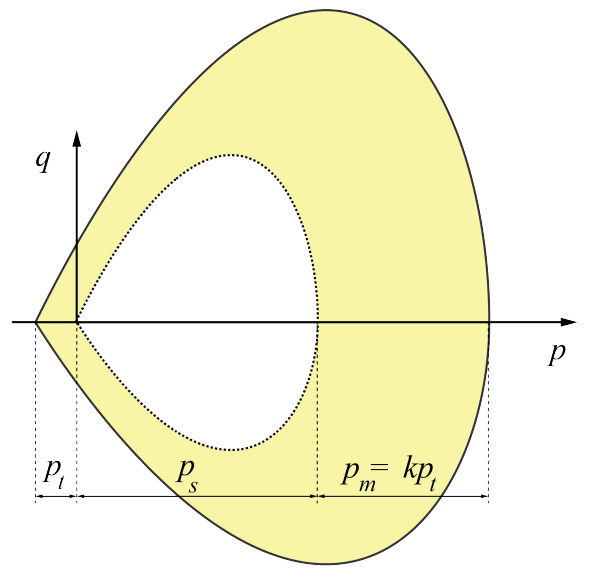

(a)

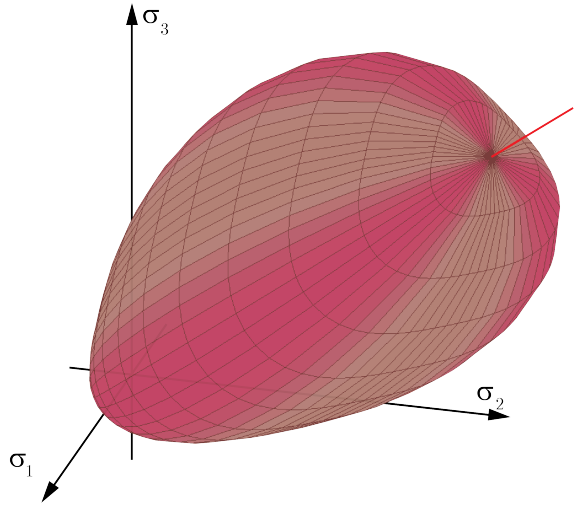

(b)

Fig. 1 Yield surface of calcarenite: a) representation in $q: p$ plane for axisymmetric compression (note: the bond-permitted stress space is outlined in grey and $p_{m}=k p_{t}$ ); b) view in principal stress space.

and the quantities $a_{f}, m_{f}, a_{g}, m_{g}$ and $k$ are material constants controlling the shape of the yield locus and of the plastic potential in the meridian $(q: p$, at constant $\theta$ ) plane.

In eqs. (33) and (34), the functions $M_{\alpha}=M_{\alpha}(\theta)$, which control the shape of the yield surface and plastic potential in the deviatoric plane, are taken from van Eekelen [17].

Non-associativity of the plastic flow is accounted for by requiring the constants $a_{f}, m_{f}$ and the function $M_{f}(\theta)$ to be different from the corresponding constants entering in the definition of $g$.

An illustration of the yield surface in the $q: p$ plane and in the principal (constitutive) stress space is given in Fig. 1. The internal variable $p_{s}$ plays the role of the preconsolidation pressure, as in classical critical-state models, and it is assumed to depend on soil microstructure in terms of grain arrangement and interactions (fabric) only. In a completely unbonded state, $p_{s}$ is the only hardening variable entering in the constitutive equation. The role of the bonding-related internal variable $p_{t}-$ which quantifies the mechanical effects of bonding in terms of the development of a nonzero tensile strength and a net increase in the isotropic yield stress - is clearly shown in Fig. 1(a).

\subsection{Generalized hardening laws}

For the given set of internal variables and the choice made for the environmental process variables, for the Ciantia and di Prisco model eq. (9) specializes into:

$$
\left\{\begin{array}{l}
\dot{p}_{s} \\
\dot{p}_{t}
\end{array}\right\}=\dot{\gamma}\left\{\begin{array}{l}
h_{s} \\
h_{t}
\end{array}\right\}+\left\{\begin{array}{ll}
N_{s w} & N_{s \xi} \\
N_{t w} & N_{t \xi}
\end{array}\right\}\left\{\begin{array}{l}
\dot{S}_{r} \\
\dot{\xi}_{d}
\end{array}\right\}
$$


The plastic hardening functions $h_{s}$ and $h_{t}$, controlling the effects of accumulated plastic deformations, have been defined as in [42] and [31]:

$$
\begin{aligned}
& h_{s}=\rho_{s} p_{s}\left\{\operatorname{tr} \boldsymbol{Q}+\zeta_{s} \sqrt{\frac{2}{3}}\|\operatorname{dev} \boldsymbol{Q}\|\right\} \\
& h_{t}=-\rho_{t} p_{t}\left\{|\operatorname{tr} \boldsymbol{Q}|+\zeta_{t} \sqrt{\frac{2}{3}}\|\operatorname{dev} \boldsymbol{Q}\|\right\}
\end{aligned}
$$

where $\rho_{s}, \rho_{t}, \zeta_{s}$ and $\zeta_{t}$ are material constants controlling the hardening rate and the relative balance between volumetric and deviatoric contributions of plastic strain rates to the evolution of $p_{s}$ and $p_{t}$.

In the definition of environmental hardening, Ciantia and di Prisco have assumed that the effects of suction hardening on the solid skeleton of the calcarenite can be considered negligible, due to the relatively large pore size and high depositional and diagenetic bond strength. In addition, the effects of chemical degradation (including particle dissolution) are taken into account by the changes in $p_{t}$ and the additional chemically-induced contribution to the deformation rate $\left(\dot{\boldsymbol{\epsilon}}^{c}\right.$, see following Sect. 3.6). These two assumptions imply:

$$
N_{s w}=0 \quad N_{s \xi}=0
$$

The remaining components of the hardening function $\boldsymbol{N}$ are given by the following expressions:

$$
N_{t w}=-\frac{p_{t}}{\left(1-\mathcal{D}_{d}\right)} \frac{\partial \mathcal{D}_{d}}{\partial S_{r}} \quad N_{t \xi}=-\frac{p_{t}}{\left(1-\mathcal{D}_{d}\right)} \frac{\partial \mathcal{D}_{d}}{\partial \xi_{d}}
$$

in terms of a unique debonding function $\mathcal{D}_{d}=\mathcal{D}_{d}\left(S_{r}, \xi_{d}\right)$.

\subsection{Environmental debonding and damage functions}

The debonding function $\mathcal{D}_{d}$ adopted by Ciantia and di Prisco has been obtained by the upscaling of a heuristic micromechanical model for the chemical degradation process at the REV scale, see [10].

By linking the reduction in bond strength to the progressive reduction in bond section caused by both STD and LTD processes and upscaling the microscopic result to the macroscopic, phenomenological level, Ciantia and di Prisco proposed the following expression for $\mathcal{D}_{d}\left(S_{r}, \xi_{d}\right)$ :

$$
\mathcal{D}_{d}= \begin{cases}1-R_{c} F\left(\Xi_{d}\right)-\left(1-R_{c}\right) F\left(\Sigma_{r}\right) & \text { if } \Sigma_{r} \leq 1 \text { and } \Xi_{d} \leq 1 \\ 1-R_{c} F\left(\Xi_{d}\right) & \text { if } \Sigma_{r}>1 \text { and } \Xi_{d} \leq 1 \\ 1-\left(1-R_{c}\right) F\left(\Sigma_{r}\right) & \text { if } \Sigma_{r} \leq 1 \text { and } \Xi_{d}>1 \\ 1 & \text { if } \Sigma_{r}>1 \text { and } \Xi_{d}>1\end{cases}
$$

where:

$$
F(x)=1-2 x+x^{2}=(1-x)^{2}
$$




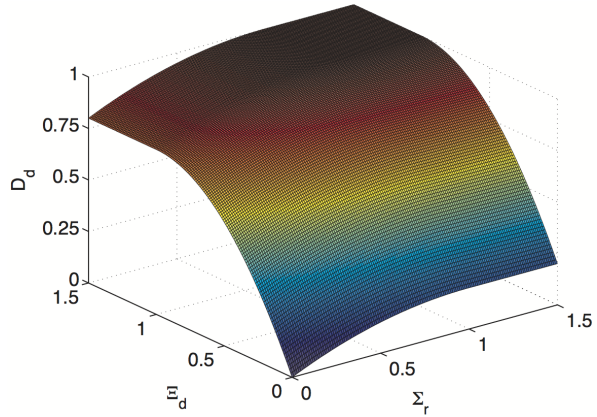

(a)

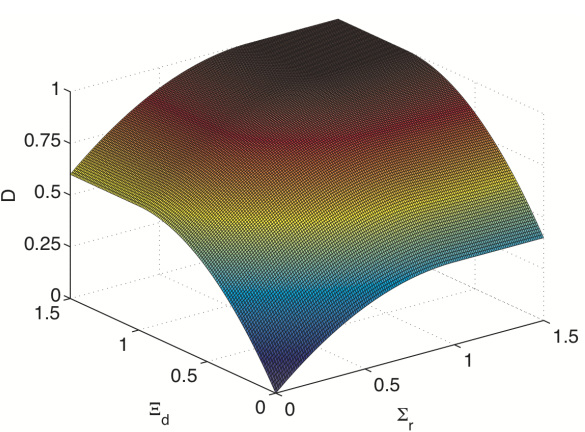

(b)

Fig. 2 Three-dimensional representation of the functions: a) $\mathcal{D}_{d}\left(\Sigma_{r}, \Xi_{d}\right)$ for $R_{c}=0.8$; b) $\mathcal{D}\left(\Sigma_{r}, \Xi_{d}\right)$ for $R_{E}=0.6$.

and:

$$
R_{c}:=\frac{\sigma_{c 0}^{w}}{\sigma_{c 0}^{d}} \quad \Xi_{d}:=\frac{\xi_{d}}{\xi_{d, \mathrm{crit}}} \quad \Sigma_{r}:=\frac{S_{r}}{S_{r, \mathrm{crit}}}
$$

In eq. (44) $1, \sigma_{c 0}^{w}$ and $\sigma_{c 0}^{d}$ represent the uniaxial compression strengths of calcarenite rock under wet and dry conditions, respectively. The quantities $\Xi_{d}$ and $\Sigma_{r}$ defined by eq. (44) $)_{2,3}$ are normalized values of $\xi_{d}$ and $S_{r}$ with respect to their respective critical values, $\xi_{d, \text { crit }}$ and $S_{r, \text { crit }}$, marking the transition between different stages of the debonding process. The first quantity represents the (normalized) amount of mass to be dissolved in order to completely eliminate diagenetic bonds; the second is the degree of saturation in correspondence of which all depositional bonds are lost. When $\Sigma_{r}$ and $\Xi_{d}$ are both greater than 1, the rock is degraded to an unbounded granular material. The function $\mathcal{D}_{d}\left(\Sigma_{r}, \Xi_{d}\right)$ is represented graphically in Fig. 2(a), for $R_{c}=0.8$.

Starting from similar micromechanical considerations at the REV scale supported by macroscopic phenomenological observations [10], Ciantia and di Prisco adopted the following damage function $\mathcal{D}$ for the free energy function of eq. (25), quite similar to eq. (42):

$$
\mathcal{D}= \begin{cases}1-R_{E} F\left(\Xi_{d}\right)-\left(1-R_{c}\right) F\left(\Sigma_{r}\right) & \text { if } \Sigma_{r} \leq 1 \text { and } \Xi_{d} \leq 1 \\ 1-R_{E} F\left(\Xi_{d}\right) & \text { if } \Sigma_{r}>1 \text { and } \Xi_{d} \leq 1 \\ 1-\left(1-R_{E}\right) F\left(\Sigma_{r}\right) & \text { if } \Sigma_{r} \leq 1 \text { and } \Xi_{d}>1 \\ 1 & \text { if } \Sigma_{r}>1 \text { and } \Xi_{d}>1\end{cases}
$$

where the function $F\left(\Sigma_{r}\right)$ is given by eq. (43), and:

$$
R_{E}:=\frac{E_{0}^{w}}{E_{0}^{d}}
$$

where $E_{0}^{d}$ and $E_{0}^{w}$ are the Young's moduli of the intact material in dry and wet states, respectively. The function $\mathcal{D}\left(\Sigma_{r}, \Xi_{d}\right)$ is represented graphically in Fig. 2(b), for $R_{E}=0.6$.

An experimental validation for the functions $\mathcal{D}_{d}$ and $\mathcal{D}$ for Gravina and Gallipoli calcarenites is provided in ref. [10]. 
3.6 Chemical deformation rate

The last aspect of the Ciantia and di Prisco model to be detailed concerns the evaluation of chemical deformations induced by the reduction in grain size associated with grain dissolution. Micromechanical considerations at the REV scale suggest that no chemical deformation can occur until the diagenetic bonds are completely dissolved $\left(\Xi_{d} \leq 1\right)$. Further increase of $\xi_{d}$ causes a progressive mass loss of the grains, which therefore reduce in size. The progressive reduction of intergranular distance which results from this process is associated to a macroscopic reduction in volume of the solid skeleton, and the appearance of a chemical deformation $\epsilon^{c}$, which increases with increasing $\xi_{d}$, i.e., with increasing mass loss.

In the simplest possible case, the chemical deformation tensor can be assumed to be isotropic, and its rate linked to the rate of change of $\xi_{d}$ by the simple relation [10]:

$$
\dot{\boldsymbol{\epsilon}}^{c}=C\left(\xi_{d}\right) \dot{\xi}_{d} \mathbf{1}
$$

which could be recast in the following format, more convenient for further developments:

$$
\dot{\boldsymbol{\epsilon}}^{c}=\mathbf{1}(\gamma \cdot \dot{\boldsymbol{\alpha}})=\boldsymbol{\Gamma} \dot{\boldsymbol{\alpha}} \quad \boldsymbol{\Gamma}=\mathbf{1} \otimes \gamma \quad \gamma=\left\{\begin{array}{c}
0 \\
C\left(\xi_{d}\right)
\end{array}\right\}
$$

In eq. (47), the scalar function $C\left(\xi_{d}\right)$ is provided by the following smoothed step function:

$$
C\left(\xi_{d}\right)= \begin{cases}0 & \text { if } \Xi_{d} \leq 1-\Delta_{d} ; \\ C_{\max }\{1-\cos \beta\} & \text { if } 1-\Delta_{d}<\Xi_{d} \leq 1+\Delta_{d} \\ C_{\max } & \text { if } \Xi_{d}>1+\Delta_{d} .\end{cases}
$$

where:

$$
\beta:=\left(\frac{\Xi_{d}-1+\Delta_{d}}{2 \Delta_{d}}\right) \pi
$$

$\Xi_{d}$ is defined by eq. $(44)_{2}$ and $\Delta_{d}$ is a model constant. The function $C$ in eq. (48) is equal to zero when diagenetic bonds are still present, and assumes the value

$C_{\max }$ when the material is fully unbonded. The constant $\Delta_{d}$ defines a transition zone $\left[1-\Delta_{d}, 1+\Delta_{d}\right]$ around the point $\Xi_{d}=1$ so that $C$ varies continuously between these two limiting values. Although very simple, eq. (47) reproduces quite satisfactorily the available experimental data [8].

For the developments discussed in the following Sect. 4, the integral of the function $C$, given by:

$$
\begin{aligned}
& I_{d}\left(\Xi_{d}\right)=\int_{0}^{\Xi_{d}} C(x) d x \text { if } \Xi_{d} \leq 1-\Delta_{d} ; \\
& \quad= \begin{cases}0 & \text { if } 1-\Delta_{d}<\Xi_{d} \leq 1+\Delta_{d} ; \\
\frac{1}{\pi} C_{\max } \Delta_{d}\{\beta-\sin \beta\} & \text { if } \Xi_{d}>1+\Delta_{d} . \\
C_{\max }\left(\Xi_{d}-1\right) & \end{cases}
\end{aligned}
$$




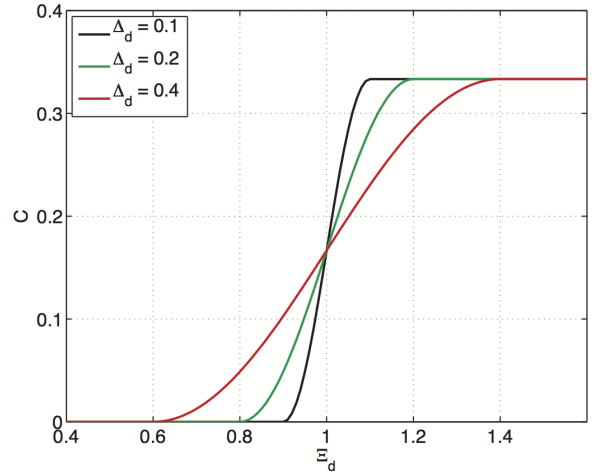

(a)

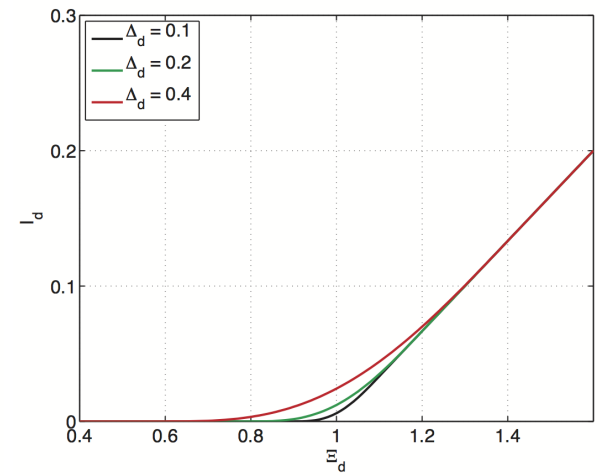

(b)

Fig. 3 Functions $C\left(\Xi_{d}\right)$ (a) and $I_{d}\left(\Xi_{d}\right)$ (b) plotted for different values of $\Delta_{d}$ and for $C_{\max }$ $=1 / 3$.

will also be useful. The two functions $C\left(\Xi_{d}\right)$ and $I_{d}\left(\Xi_{d}\right)$ are represented graphically

in Fig. 3, for different values of $\Delta_{d}$.

The presence of a chemical deformation rate proportional to the rate of the second environmental process variable gives rise to a second coupling contribution to the elastic constitutive equation, which reads:

$$
\boldsymbol{M}_{c}=-\boldsymbol{D}^{e} \boldsymbol{\Gamma}
$$

Collecting the contributions due to elastic damage and grain dissolution - eqs. (28) and $(50)_{1}$ - the coupling matrix $\boldsymbol{M}$ is finally obtained as:

$$
\boldsymbol{M}=\boldsymbol{M}_{d}+\boldsymbol{M}_{c}=-\delta_{b} \frac{\partial \psi_{0}}{\partial \boldsymbol{\epsilon}^{e}} \otimes \frac{\partial \mathcal{D}}{\partial \boldsymbol{\alpha}}-\boldsymbol{D}^{e} \boldsymbol{\Gamma}
$$

\section{Numerical integration of constitutive equations in rate-form}

For the application of constitutive models developed within the framework of plasticity with generalized hardening to practical engineering problems, a suitable computational strategy must be defined to integrate numerically the evolution equations (16) and (17) in time, given the initial state of the material and the strain and environmental process variables histories.

In the following, a specific implementation of implicit return mapping algorithms - the Generalized Backward Euler (GBE) algorithm proposed by Tamagnini et al. [42] for bonded geomaterials subject to mechanical and chemical degradation

- has been considered in this work, extending the original version of the algorithm to account for such features as elastic damage and chemical deformations.

4.1 Operator split and product formula algorithm

Considering that the hyperelastic constitutive equation (26) provides $\boldsymbol{\sigma}$ as a given function of $\epsilon^{e}$ and $\boldsymbol{\alpha}$, the constitutive stress $\boldsymbol{\sigma}$ can be replaced by the elastic strain 
Table 1 Chemo-elastic/plastic operator split of the evolution equations.

\begin{tabular}{ccc}
\hline & Step \#1 & Step \#2 \\
& Chemo-elastic predictor & Plastic corrector \\
\hline \hline$\dot{\boldsymbol{\epsilon}}=\nabla^{s}(\dot{\boldsymbol{u}})$ & $\dot{\boldsymbol{\epsilon}}=\nabla^{s}(\dot{\boldsymbol{u}})$ & $\dot{\boldsymbol{\epsilon}}=\mathbf{0}$ \\
$\dot{\boldsymbol{\epsilon}}^{c}=\boldsymbol{\Gamma} \dot{\boldsymbol{\alpha}}$ & $\dot{\boldsymbol{\epsilon}}^{c}=\boldsymbol{\Gamma} \dot{\boldsymbol{\alpha}}$ & $\dot{\boldsymbol{\epsilon}}^{c}=\mathbf{0}$ \\
$\dot{\boldsymbol{\epsilon}}^{e}=\dot{\boldsymbol{\epsilon}}-\dot{\gamma} \boldsymbol{Q}-\dot{\boldsymbol{\epsilon}}^{c}$ & $\dot{\boldsymbol{\epsilon}}^{e}=\dot{\boldsymbol{\epsilon}}-\dot{\boldsymbol{\epsilon}}^{c}$ & $\dot{\boldsymbol{\epsilon}}^{e}=-\dot{\boldsymbol{Q}}$ \\
$\dot{\boldsymbol{q}}=\dot{\gamma} \boldsymbol{h}+\boldsymbol{N} \dot{\boldsymbol{\alpha}}$ & $\dot{\boldsymbol{q}}=\boldsymbol{N} \dot{\boldsymbol{\alpha}}$ & $\dot{\boldsymbol{q}}=\dot{\gamma} \boldsymbol{h}$ \\
$\boldsymbol{\epsilon}^{e}\left(t_{n}\right)=\boldsymbol{\epsilon}_{n}^{e}$ & $\boldsymbol{\epsilon}^{e}\left(t_{n}\right)=\boldsymbol{\epsilon}_{n}^{e}$ & $\left.\boldsymbol{\epsilon}^{e}\right|_{\Delta \gamma=0}=\boldsymbol{\epsilon}_{n+1}^{e, \mathrm{tr}}$ \\
$\boldsymbol{q}\left(t_{n}\right)=\boldsymbol{q}_{n}$ & $\boldsymbol{q}\left(t_{n}\right)=\boldsymbol{q}_{n}$ & $\left.\boldsymbol{q}\right|_{\Delta \gamma=0}=\boldsymbol{q}_{n+1}^{\mathrm{tr}}$ \\
\hline
\end{tabular}

$\boldsymbol{\epsilon}^{e}$ in the set of state variables. In such a strain space formulation, the update of the state variables is obtained through the numerical integration of the following evolution equations:

$$
\begin{gathered}
\dot{\boldsymbol{\epsilon}}^{e}=\dot{\boldsymbol{\epsilon}}-\dot{\boldsymbol{\epsilon}}^{p}-\dot{\boldsymbol{\epsilon}}^{c} \\
\dot{\boldsymbol{\epsilon}}^{p}=\dot{\gamma} \boldsymbol{Q}(\boldsymbol{\sigma}, \boldsymbol{q}) \\
\dot{\boldsymbol{\epsilon}}^{c}=\boldsymbol{\Gamma}(\boldsymbol{\alpha}) \dot{\boldsymbol{\alpha}} \\
\dot{\boldsymbol{q}}=\dot{\gamma} \boldsymbol{h}(\boldsymbol{\sigma}, \boldsymbol{q})+\boldsymbol{N}(\boldsymbol{\alpha}) \dot{\boldsymbol{\alpha}}
\end{gathered}
$$

subject to the Kuhn-Tucker complementarity conditions:

$$
\dot{\gamma} \geq 0 \quad f(\boldsymbol{\sigma}, \boldsymbol{q}) \leq 0 \quad \dot{\gamma} f(\boldsymbol{\sigma}, \boldsymbol{q})=0
$$

The GBE algorithm considered in this work is a two-step algorithm originating from the chemo-elastic/plastic operator split of the evolution equations shown in Tab. 1, suggested by the additive structure of eqs. (52)-(55).

Starting from the above operator split, a product formula algorithm is constructed in a standard fashion as follows. First, the elastic predictor problem is solved and a so-called trial elastic state is obtained. Then, the constraints (56) are checked for the trial state, and if they are satisfied the trial elastic state represents the correct solution of the evolution problem. On the contrary, if they are violated, consistency must be restored by solving the plastic corrector problem, for which the trial state is taken as the appropriate initial condition.

As compared to classical elastoplasticity, in the present formulation the plastic corrector problem remains unchanged, while the elastic predictor stage is modified to account for the effects of non-mechanical degradation and chemical deformations. The elastic predictor step is the only stage of the numerical procedure in which environmental degradation effects are taken into account. It is worth noting that, due to the presence of the non-mechanical hardening contribution, during the predictor step the internal variables do not necessarily remain constant, as a change in $\boldsymbol{q}$ may be induced by a change in $\boldsymbol{\alpha}$. 
4.2 Problem 1: elastic predictor

From the physical point of view, the elastic predictor problem can be derived from the original problem (52)-(55) by freezing the plastic flow (i.e., setting $\dot{\gamma}=0$ ), and taking an incremental chemo-elastic step which ignores the constraints placed on the stress state by the yield function.

Due to the simple mathematical form of the functions $\boldsymbol{N}$ and $\boldsymbol{\Gamma}$ adopted in the Ciantia and di Prisco model, the solution of this problem can be computed in closed form. In particular, the evaluation of $\boldsymbol{\epsilon}_{n+1}^{e, t r}$ reduces to a simple geometric update of the elastic strain tensor, including chemical deformations:

$$
\epsilon_{n+1}^{e, \operatorname{tr}}=\epsilon_{n}^{e}+\Delta \epsilon_{n+1}-\Delta \epsilon_{n+1}^{c}
$$

in which the chemical strain increment is provided by:

$$
\begin{aligned}
\Delta \boldsymbol{\epsilon}_{n+1}^{c}=\left\{\int_{t_{n}}^{t_{n+1}} C\left(\xi_{d}\right) \frac{d \xi_{d}}{d t} d t\right\} \mathbf{1}=\left\{\xi_{d, \mathrm{cr}} \int_{\Xi_{d, n}}^{\Xi_{d, n+1}} C(x) d x\right\} \mathbf{1} \\
=\xi_{d, \mathrm{cr}}\left(I_{d, n+1}-I_{d, n}\right) \mathbf{1}
\end{aligned}
$$

and the function $I_{d}$ is given by eq. (49).

For the internal variable $p_{s}$, eqs. (40) yield:

$$
\dot{p}_{s}=0 \quad \text { and thus: } \quad p_{s, n+1}^{\mathrm{tr}}=p_{s, n}
$$

According to eqs. (41), the evolution equation for $p_{t}$ in the trial chemo-elastic step specializes to:

$$
\dot{p}_{t}=-\frac{p_{t}}{\left(1-\mathcal{D}_{d}\right)} \frac{\partial \mathcal{D}_{d}}{\partial S_{r}} \dot{S}_{r}-\frac{p_{t}}{\left(1-\mathcal{D}_{d}\right)} \frac{\partial \mathcal{D}_{d}}{\partial \xi_{d}} \dot{\xi}_{d}=-\frac{p_{t}}{\left(1-\mathcal{D}_{d}\right)} \dot{\mathcal{D}}_{d}
$$

This equation can be trivially integrated in closed form, yielding:

$$
p_{t, n+1}^{\mathrm{tr}}=p_{t, n}\left(\frac{1-\mathcal{D}_{d, n+1}}{1-\mathcal{D}_{d, n}}\right)
$$

The stress tensor associated with the trial elastic strain is then obtained from eq. (26) by simple function evaluation:

$$
\boldsymbol{\sigma}_{n+1}^{\mathrm{tr}}:=\left\{1+\delta_{b}\left(1-\mathcal{D}_{n+1}\right)\right\} \frac{\partial \psi_{0}}{\partial \boldsymbol{\epsilon}^{e}}\left(\boldsymbol{\epsilon}_{n+1}^{e, \operatorname{tr}}\right)
$$

If:

$$
f_{n+1}^{\mathrm{tr}}:=f\left(\boldsymbol{\sigma}_{n+1}^{\mathrm{tr}}, p_{s, n+1}^{\mathrm{tr}}, p_{t, n+1}^{\mathrm{tr}}\right) \leq 0
$$

the trial state satisfies the constraints imposed by the Kuhn-Tucker conditions. The process is then declared elastic and the trial state represents the actual final state of the material. Otherwise, the plastic corrector step is necessary.

It is worth noting that, while $p_{s}$ remains constant during the predictor stage, $p_{t}$ can change due to environmental degradation effects. This change might, in turn, give rise to a violation of the constraint (56), and thus to a change in the stress state, even for processes in which no total strain changes occur. 
4.3 Problem 2: plastic corrector

If $f_{n+1}^{\mathrm{tr}}>0$, the convexity of the yield surface implies that the trial state lies outside the yield locus. The process is thus declared plastic, and consistency is restored by solving the plastic corrector problem, which takes place at fixed total and chemical strains. Since the objective of the plastic corrector stage is to map the trial state back to the yield surface, the algorithms performing such task are commonly referred to as Return Mapping Algorithms.

In the present case, the plastic corrector problem is solved numerically by integrating the corresponding system of ordinary differential equations by an implicit Backward Euler scheme, taking the trial state as the new initial condition:

$$
\begin{aligned}
& \boldsymbol{\epsilon}_{n+1}^{e}=\boldsymbol{\epsilon}_{n+1}^{e, \operatorname{tr}}-\Delta \gamma_{n+1} \boldsymbol{Q}_{n+1} \\
& \boldsymbol{q}_{n+1}=\boldsymbol{q}_{n+1}^{\mathrm{tr}}+\Delta \gamma_{n+1} \boldsymbol{h}_{n+1}
\end{aligned}
$$

420

in which $\boldsymbol{q}_{n+1}^{\mathrm{tr}}:=\left\{p_{s, n+1}^{\mathrm{tr}}, p_{t, n+1}^{\mathrm{tr}}\right\}$. As $\Delta \gamma_{n+1}>0$, the constraints (26) now reduce to:

$$
f_{n+1}=f\left(\boldsymbol{\sigma}_{n+1}, \boldsymbol{q}_{n+1}\right)=0
$$

Equations (63)-(65) represent a system of 9 non-linear algebraic equations in the 9 unknowns $\boldsymbol{\epsilon}_{n+1}^{e}, p_{s, n+1}, p_{t, n+1}, \Delta \gamma_{n+1}$, which can be solved iteratively by Newton's method. However, due to the assumption of isotropic behavior, the system of equations (63)-(65) is redundant. In fact, the number of independent elastic strain components appearing in eq. (29) can be reduced to three, thus considerably simplifying the numerical solution of the return mapping problem.

As shown in refs. [42,3], one possible way to perform such a reduction is to reformulate the return mapping problem in principal elastic strain (or stress) space. By exploiting the spectral decomposition of the symmetric second-order tensors $\boldsymbol{\epsilon}_{n+1}^{e}, \boldsymbol{\epsilon}_{n+1}^{e, \operatorname{tr}}$ and $\boldsymbol{Q}_{n+1}=(\partial g / \partial \boldsymbol{\sigma})_{n+1}$, eq. (63) transforms into:

$$
\begin{array}{r}
\sum_{A=1}^{3}\left(\epsilon_{A}^{e}\right)_{n+1} \boldsymbol{n}_{n+1}^{(A)} \otimes \boldsymbol{n}_{n+1}^{(A)}=\sum_{A=1}^{3}\left(\epsilon_{A}^{e, \operatorname{tr}}\right)_{n+1} \boldsymbol{n}_{n+1}^{(A), \operatorname{tr}} \otimes \boldsymbol{n}_{n+1}^{(A), \operatorname{tr}}- \\
\Delta \gamma_{n+1} \sum_{A=1}^{3}\left(\frac{\partial \hat{g}}{\partial \sigma_{A}}\right)_{n+1} \boldsymbol{n}_{n+1}^{(A)} \otimes \boldsymbol{n}_{n+1}^{(A)}
\end{array}
$$

in which $\boldsymbol{n}_{n+1}^{(A), \text { tr }}$ is the $A$-th unit eigenvector of $\boldsymbol{\epsilon}_{n+1}^{e, t r}$. From eq. (66), it follows at once that:

$$
\boldsymbol{n}_{n+1}^{(A)}=\boldsymbol{n}_{n+1}^{(A), \operatorname{tr}}
$$

430 and:

$$
\left(\epsilon_{A}^{e}\right)_{n+1}=\left(\epsilon_{A}^{e, \operatorname{tr}}\right)_{n+1}-\Delta \gamma_{n+1}\left(\frac{\partial \hat{g}}{\partial \sigma_{A}}\right)_{n+1}
$$

for $A=1,2$ or 3 .

It is worth noting that:

i) as the trial elastic strain is known, so are its principal directions; therefore, the only unknown quantities to be determined remain the three principal elastic strains $\left(\epsilon_{A}^{e}\right)_{n+1}$, the two internal variables $p_{s, n+1}$ and $p_{t, n+1}$ and the plastic multiplier $\Delta \gamma_{n+1}$; 
Table 2 General return mapping in principal elastic strain space.

$$
\begin{array}{|ll|}
\hline \text { 1. } & \text { Initialize: } \\
& \hat{\boldsymbol{\epsilon}}_{n+1}^{e}=\hat{\boldsymbol{\epsilon}}_{n+1}^{e, \operatorname{tr}} \quad \boldsymbol{q}_{n+1}=\boldsymbol{q}_{n+1}^{\mathrm{tr}} \quad \Delta \gamma_{n+1}=0 \\
\text { 2. } & \text { Check for convergence: } \\
& \\
& \text { IF: }\left\{\begin{array}{l}
\left\|\boldsymbol{r}_{n+1}^{\epsilon(j)}\right\|<T O L_{\epsilon} \cdot\left\|\hat{\boldsymbol{\epsilon}}_{n+1}^{e, t \mathrm{tr}}\right\| \\
\left\|\boldsymbol{r}_{n+1}^{q(j)}\right\|<T O L_{q} \cdot\left\|\boldsymbol{q}_{n+1}^{\mathrm{tr}}\right\| \\
f_{n+1}^{(j)}<T O L_{f} \cdot p_{s}^{\operatorname{tr}}
\end{array}\right\} \\
\text { 3. } & \text { Find update at local iteration }(j): \\
& \delta \boldsymbol{x}_{n+1}^{(j)}=-\left[\left(\frac{\partial \boldsymbol{R}}{\partial \boldsymbol{x}}\right)_{n+1}^{(j)}\right]_{n+1}^{-1} \boldsymbol{R}_{n+1}^{(j)} \\
\text { 4. } & \begin{array}{l}
\text { Update state variables and plastic multiplier: } \\
\boldsymbol{x}_{n+1}^{(j+1)}=\boldsymbol{x}_{n+1}^{(j)}+\delta \boldsymbol{x}_{n+1}^{(j)}
\end{array} \\
\text { 5. } & \text { Set: } j \leftarrow j+1, \text { GO TO 2. } \\
\hline
\end{array}
$$

ii) as the chemical strains are assumed to be purely volumetric, the principal trial elastic strain directions $\boldsymbol{n}_{n+1}^{(A), \operatorname{tr}}$ are independent of the environmental process variable $\boldsymbol{\alpha}$.

Introducing for convenience the following vector notation:

$$
\hat{\boldsymbol{\epsilon}}^{e}:=\left\{\begin{array}{l}
\epsilon_{1}^{e} \\
\epsilon_{2}^{e} \\
\epsilon_{3}^{e}
\end{array}\right\} \quad \hat{\boldsymbol{\epsilon}}^{e, \operatorname{tr}}:=\left\{\begin{array}{l}
\epsilon_{1}^{e, \operatorname{tr}} \\
\epsilon_{2}^{e, \operatorname{tr}} \\
\epsilon_{3}^{e, \operatorname{tr}}
\end{array}\right\} \quad \hat{\boldsymbol{\sigma}}:=\left\{\begin{array}{l}
\sigma_{1} \\
\sigma_{2} \\
\sigma_{3}
\end{array}\right\} \quad \hat{\boldsymbol{Q}}:=\left\{\begin{array}{l}
\partial \hat{g} / \partial \sigma_{1} \\
\partial \hat{g} / \partial \sigma_{2} \\
\partial \hat{g} / \partial \sigma_{3}
\end{array}\right\}
$$

the return mapping problem in principal elastic strain space can be recast as follows:

$$
\begin{aligned}
\hat{\boldsymbol{\epsilon}}_{n+1}^{e} & =\hat{\boldsymbol{\epsilon}}_{n+1}^{e, \mathrm{tr}}-\Delta \gamma_{n+1} \hat{\boldsymbol{Q}}_{n+1} \\
\boldsymbol{q}_{n+1} & =\boldsymbol{q}_{n+1}^{\mathrm{tr}}+\Delta \gamma_{n+1} \boldsymbol{h}_{n+1} \\
f_{n+1} & :=f\left(\hat{\boldsymbol{\sigma}}_{n+1}, \boldsymbol{q}_{n+1}\right)=0
\end{aligned}
$$

440 Now, let:

$$
\boldsymbol{x}_{n+1}:=\left\{\hat{\boldsymbol{\epsilon}}_{n+1}^{e T} \boldsymbol{q}_{n+1}^{T} \Delta \gamma_{n+1}\right\}^{T} \in \mathbb{R}^{6}
$$

be a 6 -dimensional vector containing the six unknowns of the problem. The return mapping equations (70)-(72) require the vanishing of the following residual vector:

$$
\boldsymbol{R}_{n+1}\left(\boldsymbol{x}_{n+1}\right):=\left\{\begin{array}{l}
\boldsymbol{r}_{n+1}^{\epsilon} \\
\boldsymbol{r}_{n+1}^{q} \\
f_{n+1}
\end{array}\right\}:=\left\{\begin{array}{c}
-\hat{\boldsymbol{\epsilon}}_{n+1}^{e}+\hat{\boldsymbol{\epsilon}}_{n+1}^{e, \mathrm{tr}}-\Delta \gamma_{n+1} \hat{\boldsymbol{Q}}_{n+1} \\
-\boldsymbol{q}_{n+1}+\boldsymbol{q}_{n+1}^{\mathrm{tr}}+\Delta \gamma_{n+1} \boldsymbol{h}_{n+1} \\
f_{n+1}
\end{array}\right\}=\mathbf{0}
$$

The steps required for the iterative solution of eq. (74) via Newton's method are outlined in table 2 .

Step 3 in the procedure requires the inversion of a $6 \times 6$ square matrix. However, by observing that the last component of the residual vector $\boldsymbol{R}_{n+1}^{(j)}$ does not depend on $\Delta \gamma_{n+1}$, the resulting linearized system of equation can be reduced in size via 
static condensation. Let the residual vector, $\boldsymbol{R}_{n+1}^{(j)}$, and the vector of unknowns, $\boldsymbol{x}_{n+1}^{(j)}$, be partitioned as follows:

$$
\boldsymbol{R}_{n+1}^{(j)}:=\left\{\begin{array}{c}
\widetilde{\boldsymbol{R}}_{n+1}^{(j)} \\
f_{n+1}^{(j)}
\end{array}\right\} ; \quad \boldsymbol{x}_{n+1}^{(j)}:=\left\{\begin{array}{c}
\widetilde{\boldsymbol{x}}_{n+1}^{(j)} \\
\Delta \gamma_{n+1}^{(j)}
\end{array}\right\} ; \quad \widetilde{\boldsymbol{x}}_{n+1}^{(j)}:=\left\{\begin{array}{c}
\hat{\boldsymbol{\epsilon}}_{n+1}^{e,(j)} \\
\boldsymbol{q}_{n+1}^{(j)}
\end{array}\right\}
$$

445 then, the tangent operator $(\partial \boldsymbol{R} / \partial \boldsymbol{x})_{n+1}^{(j)}$ can be evaluated as:

$$
\left(\frac{\partial \boldsymbol{R}}{\partial \boldsymbol{x}}\right)_{n+1}^{(j)}=\left[\begin{array}{cc}
-\boldsymbol{A} & -\boldsymbol{U} \\
\boldsymbol{V} & 0
\end{array}\right]_{n+1}^{(j)}
$$

The first $(5 \times 5)$ minor $\boldsymbol{A}$ can, in turn, be partitioned as:

$$
\boldsymbol{A}:=\left[\begin{array}{ll}
\boldsymbol{A}_{11} & \boldsymbol{A}_{12} \\
\boldsymbol{A}_{21} & \boldsymbol{A}_{22}
\end{array}\right]
$$

where:

$$
\begin{array}{rlrl}
\boldsymbol{A}_{11} & :=\boldsymbol{I}_{(3 \times 3)}+\Delta \gamma\left(\frac{\partial^{2} \hat{g}}{\partial \hat{\boldsymbol{\sigma}} \otimes \partial \hat{\boldsymbol{\sigma}}}\right) \hat{\boldsymbol{d}}^{e} & \boldsymbol{A}_{12}:=\Delta \gamma\left(\frac{\partial^{2} \hat{g}}{\partial \hat{\boldsymbol{\sigma}} \otimes \partial \boldsymbol{q}}\right) \\
\boldsymbol{A}_{21}:=-\Delta \gamma \boldsymbol{B}_{\sigma} \hat{\boldsymbol{d}}^{e} & \boldsymbol{A}_{22}:=\boldsymbol{I}_{(2 \times 2)}-\Delta \gamma \boldsymbol{B}_{q} \\
\boldsymbol{U} & :=\left\{\hat{\boldsymbol{Q}}^{T},-\boldsymbol{h}^{T}\right\}^{T} & \boldsymbol{V}:=\left\{\hat{\boldsymbol{P}}^{T} \hat{\boldsymbol{d}}^{e}, \boldsymbol{W}^{T}\right\} \\
\hat{\boldsymbol{P}} & :=\frac{\partial \hat{f}}{\partial \hat{\boldsymbol{\sigma}}} & \boldsymbol{W}:=\frac{\partial \hat{f}}{\partial \boldsymbol{q}} \\
\boldsymbol{B}_{\sigma}:=\frac{\partial \boldsymbol{h}}{\partial \hat{\boldsymbol{\sigma}}} & \boldsymbol{B}_{q}:=\frac{\partial \boldsymbol{h}}{\partial \boldsymbol{q}}
\end{array}
$$

and:

$$
\hat{\boldsymbol{d}}^{e}:=\left\{1+\delta_{b}(1-\mathcal{D})\right\} \frac{\partial^{2} \hat{\psi}}{\partial \hat{\boldsymbol{\epsilon}}^{e} \otimes \partial \hat{\boldsymbol{\epsilon}}^{e}}
$$

is the $(3 \times 3)$ elastic stiffness matrix in the principal elastic strain space.

The system of linear equations in step 3 can then be rewritten as:

$$
\begin{gathered}
\boldsymbol{A}_{n+1}^{(j)} \delta \widetilde{\boldsymbol{x}}_{n+1}^{(j)}+\boldsymbol{U}_{n+1}^{(j)} \delta \Delta \gamma_{n+1}^{(j)}=\widetilde{\boldsymbol{R}}_{n+1}^{(j)} \\
\boldsymbol{V}_{n+1}^{(j)} \delta \widetilde{\boldsymbol{x}}_{n+1}^{(j)}=-f_{n+1}^{(j)}
\end{gathered}
$$

Eqs. (84) and (85) can be solved in two stages. First, solve eq. (45) for $\delta \widetilde{\boldsymbol{x}}_{n+1}^{(j)}$, 450 substitute the result in eq. (46) and evaluate the change in consistency parameter as:

$$
\delta \Delta \gamma_{n+1}^{(j)}=\frac{1}{\left(\boldsymbol{V}\left[\boldsymbol{A}^{-1}\right] \boldsymbol{U}\right)_{n+1}^{(j)}}\left\{f_{n+1}^{(j)}+\left(\boldsymbol{V}\left[\boldsymbol{A}^{-1}\right] \widetilde{\boldsymbol{R}}\right)_{n+1}^{(j)}\right\}
$$

then, compute $\delta \widetilde{\boldsymbol{x}}_{n+1}^{(j)}$ as:

$$
\delta \widetilde{\boldsymbol{x}}_{n+1}^{(j)}=\left[\boldsymbol{A}^{-1}\right]_{n+1}^{(j)}\{\widetilde{\boldsymbol{R}}-\delta \Delta \gamma \boldsymbol{U}\}_{n+1}^{(j)}
$$


In this way, only the inversion of the $(5 \times 5)$ matrix $\boldsymbol{A}_{n+1}^{(j)}$ is required for each iteration. Although a closed form inversion of the matrix $\boldsymbol{A}_{n+1}^{(j)}$ is possible, in the actual implementation of the algorithm this task has been performed numerically, using the LAPACK library.

A classical difficulty in the application of GBE algorithms to complex, threeinvariants plasticity models lies in the need of computing the gradients of the flow rule and the hardening law. this, in turns, require the evaluation of the second gradient of the plastic potential function with respect to $\sigma$. One of the advantages associated with the formulation of the return mapping stage in the principal elastic strain space is that this task can be considerably simplified, as only the computation of the $(3 \times 3)$ matrix:

$$
\nabla_{\hat{\boldsymbol{\sigma}}} \nabla_{\hat{\boldsymbol{\sigma}}}(g)=\frac{\partial^{2} \hat{g}}{\partial \hat{\boldsymbol{\sigma}} \otimes \partial \hat{\boldsymbol{\sigma}}}
$$

is now needed.

\section{Linearization and consistent tangent operators}

In a standard finite element context, the starting point for the solution of a coupled equilibrium and conduction (of water, gas and chemical species dissolved in the pore water) problem is the weak form of the balance equations (momentum; mass of solid; mass of fluids present in the pore space; mass of chemical species dissolved in the pore space).

Once these equations are discretized in space introducing a standard FE (semidiscrete) approximation and integrated in time using a suitable time-integration algorithm, the resulting system of non-linear algebraic equations is typically solved - for each increment of the adopted time discretization - by using an iterative strategy. Provided that the nonlinear system of algebraic equations is linearizable, Newton's method represents a very effective choice for this task, due to its quadratic convergence rate.

In particular, the linearization of the weak form of the equilibrium equation with respect to the independent fields $\boldsymbol{u}$ and $\boldsymbol{\alpha}$ (at time $t_{n+1}$ ) reads:

$$
\begin{gathered}
D_{\boldsymbol{u}} G^{\text {int }}\left(\boldsymbol{u}_{n+1}^{(k)}, \boldsymbol{\alpha}_{n+1}^{(k)}, \boldsymbol{\eta}\right)\left[\delta \boldsymbol{u}_{n+1}^{(k)}\right]=\int_{\mathcal{B}}\left\{\nabla^{s} \boldsymbol{\eta} \cdot(\widetilde{\boldsymbol{D}})_{n+1}^{(k)} \nabla^{s}(\delta \boldsymbol{u})_{n+1}^{(k)}\right\} d v \\
D_{\boldsymbol{\alpha}} G^{\text {int }}\left(\boldsymbol{u}_{n+1}^{(k)}, \boldsymbol{\alpha}_{n+1}^{(k)}, \boldsymbol{\eta}\right)\left[\delta \boldsymbol{\alpha}_{n+1}^{(k)}\right]=\int_{\mathcal{B}}\left\{\nabla^{s} \boldsymbol{\eta} \cdot(\widetilde{\boldsymbol{M}})_{n+1}^{(k)} \delta \boldsymbol{\alpha}_{n+1}^{(k)}\right\} d v
\end{gathered}
$$

where:

$$
\widetilde{\boldsymbol{D}}_{n+1}^{(k)}:=\frac{\partial \boldsymbol{\sigma}_{n+1}^{(k)}}{\partial \boldsymbol{\epsilon}_{n+1}^{(k)}} \quad \widetilde{\boldsymbol{M}}_{n+1}^{(k)}:=\frac{\partial \boldsymbol{\sigma}_{n+1}^{(k)}}{\partial \boldsymbol{\alpha}_{n+1}^{(k)}}
$$

are the so-called consistent tangent stiffness and coupling matrices describing the changes of the stress tensor provided by the state update algorithm of Sect. 4 for infinitesimal changes of the unknown fields $\delta \boldsymbol{u}_{n+1}^{(k)}$ and $\delta \boldsymbol{\alpha}_{n+1}^{(k)}$ at the $(k)$-th iteration of time step $\left[t_{n}, t_{n+1}\right][41,40]$. The derivation of these two quantities is detailed in the following. 
5.1 Consistent tangent stiffness $\widetilde{\boldsymbol{D}}_{n+1}^{(k)}$

If the trial state is inside or on the yield locus, and the process is declared elastic, the consistent tangent stiffness coincides with the material elastic tangent stiffness provided by eq. (27):

$$
\widetilde{\boldsymbol{D}}_{n+1}^{(k)}=\boldsymbol{D}_{n+1}^{e,(k)}=\left\{1+\delta_{b}\left(1-\mathcal{D}_{n+1}^{(k)}\right)\right\}\left(\frac{\partial^{2} \psi_{0}}{\partial \boldsymbol{\epsilon}^{e} \otimes \partial \boldsymbol{\epsilon}^{e}}\right)_{n+1}^{(k)}
$$

480 If, on the other hand, the process is plastic, then the evaluation of $\widetilde{\boldsymbol{D}}_{n+1}^{(k)}$ can be performed exploiting a result by Ogden [33].

Omitting the superscript $(k)$ referring to the current global iteration to ease the notation, from the spectral decomposition of $\boldsymbol{\epsilon}_{n+1}^{e, \operatorname{tr}(k)}$, the elastic trial strain rate can be expressed as:

$$
\begin{aligned}
\dot{\boldsymbol{\epsilon}}_{n+1}^{e, \operatorname{tr}}=\sum_{A=1}^{3}\left(\dot{\epsilon}_{A}\right)_{n+1}^{e, \operatorname{tr}} \boldsymbol{n}_{n+1}^{(A), \operatorname{tr}} \otimes \boldsymbol{n}_{n+1}^{(B), \operatorname{tr}} & \\
& +\sum_{A \neq B}\left(\Omega_{A B}^{e, \operatorname{tr}}\right)_{n+1}\left[\left(\epsilon_{B}^{e, \operatorname{tr}}\right)_{n+1}-\left(\epsilon_{A}^{e, \operatorname{tr}}\right)_{n+1}\right] \boldsymbol{n}_{n+1}^{(A), \operatorname{tr}} \otimes \boldsymbol{n}_{n+1}^{(B), \operatorname{tr}}
\end{aligned}
$$

where the symbol $\sum_{A \neq B}$ implies a double sum over all the indices $A, B \in\{1,2,3\}$ and $B \neq A$. In eq. $(92)$,

$$
\boldsymbol{\Omega}_{n+1}^{e, \operatorname{tr}}:=\sum_{A=1}^{3} \dot{\boldsymbol{n}}_{n+1}^{(A), \mathrm{tr}} \otimes \boldsymbol{n}_{n+1}^{(A), \mathrm{tr}}
$$

is the antisymmetric spin tensor of the principal axes of $\epsilon^{e, \operatorname{tr}}$, [33]. A similar expression holds for $\dot{\boldsymbol{\sigma}}_{n+1}$.

As the principal directions of $\boldsymbol{\sigma}_{n+1}$ and $\boldsymbol{\epsilon}_{n+1}^{e, \operatorname{tr}}$ coincide, and the principal values of the stress tensor $\left(\sigma_{A}\right)_{n+1}$ depend only on $\left(\epsilon_{B}\right)_{n+1}^{e, \text { tr }}$ (with $B=1,2$ or 3 ) via the return mapping equations $(70)-(72)$, it follows that:

$$
\begin{aligned}
\dot{\boldsymbol{\sigma}}_{n+1}=\sum_{A, B=1}^{3} \frac{\partial\left(\sigma_{A}\right)_{n+1}}{\partial\left(\epsilon_{B}^{e, t \mathrm{tr}}\right)_{n+1}}\left(\dot{\epsilon}_{B}^{e, \operatorname{tr}}\right)_{n+1} \boldsymbol{n}_{n+1}^{(A), \operatorname{tr}} \otimes \boldsymbol{n}_{n+1}^{(A), \operatorname{tr}} \\
+\sum_{A \neq B}\left\{\left(\Omega_{A B}^{e, \operatorname{tr}}\right)_{n+1}\left[\left(\epsilon_{B}^{e, \operatorname{tr}}\right)_{n+1}-\left(\epsilon_{A}^{e, \operatorname{tr}}\right)_{n+1}\right]\right. \\
\left.\times\left[\frac{\left(\sigma_{B}\right)_{n+1}-\left(\sigma_{A}\right)_{n+1}}{\left(\epsilon_{B}^{e, \operatorname{tr}}\right)_{n+1}-\left(\epsilon_{A}^{e, \operatorname{tr}}\right)_{n+1}}\right] \boldsymbol{n}_{n+1}^{(A), \operatorname{tr}} \otimes \boldsymbol{n}_{n+1}^{(B), \operatorname{tr}}\right\}
\end{aligned}
$$

A direct comparison between eqs. (92) and (94) then yields:

$$
\begin{aligned}
\widetilde{\boldsymbol{D}}_{n+1}^{(k)}=\sum_{A, B=1}^{3}\left(\hat{d}_{A B}\right)_{n+1}^{(k)} \boldsymbol{m}_{n+1}^{(A), \operatorname{tr}} & \otimes \boldsymbol{m}_{n+1}^{(B), \operatorname{tr}} \\
+ & \frac{1}{2} \sum_{A \neq B} \frac{\left(\sigma_{B}\right)_{n+1}^{(k)}-\left(\sigma_{A}\right)_{n+1}^{(k)}}{\left(\epsilon_{B}^{e, \operatorname{tr}}\right)_{n+1}^{(k)}-\left(\epsilon_{A}^{e, t r}\right)_{n+1}^{(k)}} \boldsymbol{\mu}_{n+1}^{(A B), \operatorname{tr}}
\end{aligned}
$$


where:

$$
\begin{gathered}
\left(\hat{d}_{A B}\right)_{n+1}^{(k)}:=\frac{\partial\left(\sigma_{A}\right)_{n+1}^{(k)}}{\partial\left(\epsilon_{B}^{e, t r}\right)_{n+1}^{(k)}}=\frac{\partial\left(\sigma_{A}\right)_{n+1}^{(k)}}{\partial\left(\epsilon_{B}\right)_{n+1}^{(k)}} \quad(A, B=1,2,3) \\
\boldsymbol{\mu}_{n+1}^{(A B), \operatorname{tr}}:=\boldsymbol{m}_{n+1}^{(A B), \operatorname{tr}} \otimes \boldsymbol{m}_{n+1}^{(A B), \operatorname{tr}}+\boldsymbol{m}_{n+1}^{(A B), \operatorname{tr}} \otimes \boldsymbol{m}_{n+1}^{(B A), \operatorname{tr}} \\
\boldsymbol{m}_{n+1}^{(A B), \operatorname{tr}}:=\boldsymbol{n}_{n+1}^{(A), \operatorname{tr}} \otimes \boldsymbol{n}_{n+1}^{(B), \operatorname{tr}}
\end{gathered}
$$

Eq. (95) becomes singular in presence of repeated eigenvalues, due to the term $\left[\left(\sigma_{B}\right)_{n+1}-\left(\sigma_{A}\right)_{n+1}\right] /\left[\left(\epsilon_{B}^{e, \operatorname{tr}}\right)_{n+1}-\left(\epsilon_{A}^{e, \operatorname{tr}}\right)_{n+1}\right]$ in the second sum on the RHS. As pointed out by Ogden [33], this singularity can be solved by observing that, in presence of repeated eigenvalues:

$$
\frac{\sigma_{B}-\sigma_{A}}{\epsilon_{B}^{e, \operatorname{tr}}-\epsilon_{A}^{e, t \mathrm{tr}}} \quad \text { should be replaced by } \quad \frac{\partial}{\partial \epsilon_{B}^{e, t \mathrm{tr}}}\left(\sigma_{B}-\sigma_{A}\right)=\hat{d}_{B B}-\hat{d}_{A B}
$$

whenever $\epsilon_{B}^{e, t r}=\epsilon_{A}^{e, t r}$. As remarked in [39], although closed-form solutions are available for the eigenvectors of a symmetric second order tensor, algorithmic iterative solutions are usually preferable to avoid difficulties associated with repeated roots. In this work, the LAPACK library has been used for this purpose.

5.2 Consistent tangent stiffness matrix $\hat{\boldsymbol{d}}_{n+1}^{(k)}$

The linearization procedure outlined in the previous section requires the evaluation of the matrix of consistent tangent moduli referred to the principal axes of stress and elastic strain defined in eq. (96). This matrix is strongly dependent on the particular structure of the constitutive equations and on the stress-point algorithm employed for their integration.

To compute it in closed form, we first observe that, from the free energy function of eq. (25), the infinitesimal stress variation associated to a perturbation of the displacement field at constant $\boldsymbol{\alpha}$ is given by:

$$
d \hat{\boldsymbol{\sigma}}=\left\{1+\delta_{b}(1-\mathcal{D})\right\} \frac{\partial^{2} \hat{\psi}_{0}}{\partial \hat{\boldsymbol{\epsilon}}^{e} \otimes \partial \hat{\boldsymbol{\epsilon}}^{e}} d \hat{\boldsymbol{\epsilon}}^{e}=\hat{\boldsymbol{d}}^{e} d \hat{\boldsymbol{\epsilon}}^{e}
$$

where the subscript $(n+1)$ and the superscript $(k)$ have been omitted to ease the notation. In eq. (99), the vector $d \hat{\boldsymbol{\epsilon}}^{e}$ represents the infinitesimal change of $\hat{\boldsymbol{\epsilon}}^{e}$ induced in the solution of the integration algorithm by a given infinitesimal change of the total principal strains $d \hat{\boldsymbol{\epsilon}}$.

This quantity can be obtained by differentiating eqs. (70)-(72) for a given $d \hat{\boldsymbol{\epsilon}}=d \hat{\boldsymbol{\epsilon}}^{e, \text { tr }}$ and $d \boldsymbol{\alpha}=\mathbf{0}$. Adopting the notations introduced in eq. $(75)_{3}$ and eqs. (77) to (82), the differential form of eqs. (70) and (71) reads:

$$
\boldsymbol{A} d \widetilde{\boldsymbol{x}}=\boldsymbol{T} d \hat{\boldsymbol{\epsilon}}^{e, \operatorname{tr}}-\boldsymbol{U} d(\Delta \gamma)=\boldsymbol{T} d \hat{\boldsymbol{\epsilon}}-\boldsymbol{U} d(\Delta \gamma)
$$

in which $\boldsymbol{T}$ is a $(5 \times 3)$ matrix given by:

$$
\boldsymbol{T}:=\left[\begin{array}{lll}
1 & 0 & 0 \\
0 & 1 & 0 \\
0 & 0 & 1 \\
0 & 0 & 0 \\
0 & 0 & 0
\end{array}\right]
$$


The increment of the plastic multiplier associated with a change of total strains can be determined by differentiating the consistency condition (72):

$$
d f=\hat{\boldsymbol{P}}^{T} \hat{\boldsymbol{d}}^{e} d \hat{\boldsymbol{\epsilon}}^{e}+\boldsymbol{W}^{T} d \boldsymbol{q}=\boldsymbol{V} d \widetilde{\boldsymbol{x}}=0
$$

Solving eq. (104) for $d \widetilde{\boldsymbol{x}}$ and substituting the result in eq. (102), the following

expression is obtained for $d(\Delta \gamma)$ :

$$
d(\Delta \gamma)=\frac{1}{\left(\boldsymbol{V} \boldsymbol{A}^{-1} \boldsymbol{U}\right)} \boldsymbol{V} \boldsymbol{A}^{-1} \boldsymbol{T} d \hat{\boldsymbol{\epsilon}}
$$

This, in turns, provides the final expressions for $d \widetilde{\boldsymbol{x}}$ :

$$
d \widetilde{\boldsymbol{x}}=\left[\boldsymbol{A}^{-1}-\frac{\boldsymbol{A}^{-1} \boldsymbol{U} \boldsymbol{V} \boldsymbol{A}^{-1}}{\left(\boldsymbol{V} \boldsymbol{A}^{-1} \boldsymbol{U}\right)}\right] \boldsymbol{T} d \hat{\boldsymbol{\epsilon}}
$$

Since $d \hat{\boldsymbol{\epsilon}}^{e}=\boldsymbol{T}^{T} d \widetilde{\boldsymbol{x}}$, it follows that:

$$
d \hat{\boldsymbol{\epsilon}}^{e}=\boldsymbol{\Xi} d \hat{\boldsymbol{\epsilon}} \quad \boldsymbol{\Xi}:=\boldsymbol{T}^{T}\left[\boldsymbol{A}^{-1}-\frac{\boldsymbol{A}^{-1} \boldsymbol{U} \boldsymbol{V} \boldsymbol{A}^{-1}}{\left(\boldsymbol{V} \boldsymbol{A}^{-1} \boldsymbol{U}\right)}\right] \boldsymbol{T}
$$

Substituting eq. (105) in eq. (99), we obtain finally:

$$
\hat{\boldsymbol{d}}_{n+1}^{(k)}:=\hat{\boldsymbol{d}}_{n+1}^{e(k)} \boldsymbol{\Xi}_{n+1}^{(k)}
$$

5.3 Consistent tangent coupling matrix $\widetilde{\boldsymbol{M}}_{n+1}^{(k)}$

If the trial state is inside the yield locus, the consistent tangent coupling matrix

coincides with the coupling damage matrix $\boldsymbol{M}_{\boldsymbol{d}}$ provided by eq. (28):

$$
\widetilde{\boldsymbol{M}}_{n+1}^{(k)}=\left(\boldsymbol{M}_{d}\right)_{n+1}^{(k)}
$$

If, on the other hand, the process is plastic, the spectral decomposition of the stress tensor $\boldsymbol{\sigma}$ provides the following expression for the consistent tangent coupling matrix:

$$
\widetilde{\boldsymbol{M}}_{n+1}^{(k)}=\frac{\partial}{\partial \boldsymbol{\alpha}_{n+1}^{(k)}}\left\{\sum_{A=1}^{3}\left(\sigma_{A}\right)_{n+1}^{(k)} \boldsymbol{m}_{n+1}^{(A), \operatorname{tr}}\right\}=\sum_{A=1}^{3} \boldsymbol{m}_{n+1}^{(A), \operatorname{tr}} \otimes \frac{\partial\left(\sigma_{A}\right)_{n+1}^{(k)}}{\partial \boldsymbol{\alpha}_{n+1}^{(k)}}
$$

where the last results is a consequence of the independence of the principal trial strain directions on the environmental process variable $\boldsymbol{\alpha}$, see Sect. 4.3.

Adopting Voigt notation for the symmetric second order tensors $\boldsymbol{\sigma}$ and $\boldsymbol{m}^{A}$, eq. (108) can be rewritten in terms of the following matrix product:

$$
\widetilde{\boldsymbol{M}}_{n+1}^{(k)}=\widehat{\boldsymbol{m}}_{n+1}^{(k)} \frac{\partial \hat{\boldsymbol{\sigma}}_{n+1}^{(k)}}{\partial \boldsymbol{\alpha}_{n+1}^{(k)}} \quad \widehat{\boldsymbol{m}}_{n+1}^{(k)}=\left[\boldsymbol{m}_{n+1}^{(1), \mathrm{tr}} \boldsymbol{m}_{n+1}^{(2), \mathrm{tr}} \boldsymbol{m}_{n+1}^{(3), \mathrm{tr}}\right]
$$

The problem is thus reduced to the calculation of the $(3 \times 2)$ matrix of the derivative of principal stress components with respect to the two environmental process 
variables. As in previous Sect. 5.2, this is obtained by linearizing the eqs. (70)(72) for an infinitesimal perturbation of $\boldsymbol{\alpha}$ at constant total strain. In the following derivations, the subscript $(n+1)$ and the superscript $(k)$ will be omitted to ease the notation.

In a plastic process, a variation of $\boldsymbol{\alpha}$ at constant total deformation can affect elastic strains by inducing plastic deformations to restore consistency. Therefore, the variation in principal stresses associated to an infinitesimal change $d \boldsymbol{\alpha}$ at $d \hat{\boldsymbol{\epsilon}}=$ $\mathbf{0}$ can be expressed as:

$$
d \hat{\boldsymbol{\sigma}}=\hat{\boldsymbol{M}}_{d} d \boldsymbol{\alpha}+\hat{\boldsymbol{d}}^{e} d \hat{\boldsymbol{\epsilon}}^{e}
$$

with:

$$
\hat{\boldsymbol{M}}_{d}=-\delta_{b} \frac{\partial \hat{\psi}_{0}}{\partial \hat{\boldsymbol{\epsilon}}^{e}} \otimes \frac{\partial \mathcal{D}}{\partial \boldsymbol{\alpha}}
$$

and $\hat{\boldsymbol{d}}^{e}$ given by eq. (83).

The rate of principal elastic strain $d \hat{\boldsymbol{\epsilon}}^{e}$ appearing in eq. (110) can be obtained by differentiating eqs. (70)-(72), considering that, in this case, the trial values of elastic strain $\hat{\boldsymbol{\epsilon}}^{e, \operatorname{tr}}$ and internal variables $\boldsymbol{q}^{\text {tr }}$ depend on $\boldsymbol{\alpha}$ via eqs. (57), (59) and (61), so that:

$$
d \hat{\boldsymbol{\epsilon}}^{e, \operatorname{tr}}=-d \hat{\boldsymbol{\epsilon}}^{c}=-\hat{\boldsymbol{\Gamma}} d \boldsymbol{\alpha} \quad d \boldsymbol{q}^{\mathrm{tr}}=\boldsymbol{N} d \boldsymbol{\alpha}
$$

where:

$$
\hat{\boldsymbol{\Gamma}}=\hat{\boldsymbol{\delta}} \boldsymbol{\gamma}^{T} \hat{\boldsymbol{\delta}}=\left\{\begin{array}{lll}
1 & 1 & 1
\end{array}\right\}^{T}
$$

and $\gamma$ and $\boldsymbol{N}$ given by eqs. (47) 3 and (37).

Adopting the notations introduced in eq. $(75)_{3}$ and eqs. (77) to (82), the differential form of eqs. (70) and (71) reads:

$$
\boldsymbol{A} d \widetilde{\boldsymbol{x}}=\boldsymbol{C} d \boldsymbol{\alpha}-\boldsymbol{U} d(\Delta \gamma)
$$

in which:

$$
C=\left\{\begin{array}{c}
-\Gamma^{*} \\
N^{*}
\end{array}\right\}
$$

and:

$$
\boldsymbol{\Gamma}^{*}:=\boldsymbol{\Gamma}+\Delta \gamma\left(\frac{\partial^{2} \hat{g}}{\partial \hat{\boldsymbol{\sigma}} \otimes \partial \hat{\boldsymbol{\sigma}}}\right) \hat{\boldsymbol{M}}_{d} \quad \boldsymbol{N}^{*}:=\boldsymbol{N}+\Delta \gamma \boldsymbol{B}_{\sigma} \hat{\boldsymbol{M}}_{d}
$$

The increment of the plastic multiplier associated with a change of environmental process variables can be determined by differentiating the consistency condition (72):

$$
d f=\hat{\boldsymbol{P}}^{T} \hat{\boldsymbol{M}}_{d} d \boldsymbol{\alpha}+\hat{\boldsymbol{P}}^{T} \hat{\boldsymbol{d}}^{e} d \hat{\boldsymbol{\epsilon}}^{e}+\boldsymbol{W}^{T} d \boldsymbol{q}=\boldsymbol{V} d \widetilde{\boldsymbol{x}}+\hat{\boldsymbol{P}}^{T} \hat{\boldsymbol{M}}_{d} d \boldsymbol{\alpha}=0
$$

Solving eq. (113) for $d \widetilde{\boldsymbol{x}}$ and substituting the result in eq. (116), the following expression is obtained for $d(\Delta \gamma)$ :

$$
d(\Delta \gamma)=\frac{1}{\left(\boldsymbol{V} \boldsymbol{A}^{-1} \boldsymbol{U}\right)}\left(\boldsymbol{V} \boldsymbol{A}^{-1} \boldsymbol{C}+\hat{\boldsymbol{P}}^{T} \hat{\boldsymbol{M}}_{d}\right) d \boldsymbol{\alpha}
$$


This, in turns, provides the final expressions for $d \widetilde{\boldsymbol{x}}$ :

$$
d \widetilde{\boldsymbol{x}}=\boldsymbol{\Theta} d \boldsymbol{\alpha}
$$

where:

$$
\Theta:=\boldsymbol{A}^{-1} \boldsymbol{C}-\frac{\left(\boldsymbol{A}^{-1} \boldsymbol{U}\right)\left(\boldsymbol{V} \boldsymbol{A}^{-1}\right) \boldsymbol{C}}{\left(\boldsymbol{V} \boldsymbol{A}^{-1} \boldsymbol{U}\right)}-\frac{\left(\boldsymbol{A}^{-1} \boldsymbol{U}\right)\left(\hat{\boldsymbol{P}}^{T} \hat{\boldsymbol{M}}_{d}\right)}{\left(\boldsymbol{V} \boldsymbol{A}^{-1} \boldsymbol{U}\right)}
$$

Since $d \hat{\boldsymbol{\epsilon}}^{e}=\boldsymbol{T}^{T} d \widetilde{\boldsymbol{x}}$, we have:

$$
d \hat{\boldsymbol{\epsilon}}^{e}=\boldsymbol{\Xi}_{d} d \boldsymbol{\alpha} \quad \boldsymbol{\Xi}_{d}:=\boldsymbol{T}^{T} \boldsymbol{\Theta}
$$

Substituting eq. (120) in eq. (110), we obtain finally:

$$
\frac{\partial \hat{\boldsymbol{\sigma}}_{n+1}^{(k)}}{\partial \boldsymbol{\alpha}_{n+1}^{(k)}}=\left(\hat{\boldsymbol{M}}_{d}+\hat{\boldsymbol{d}}^{e} \boldsymbol{\Xi}_{d}\right)_{n+1}^{(k)}
$$

and:

$$
\widetilde{\boldsymbol{M}}_{n+1}^{(k)}=\widehat{\boldsymbol{m}}_{n+1}^{(k)}\left(\hat{\boldsymbol{M}}_{d}+\hat{\boldsymbol{d}}^{e} \boldsymbol{\Xi}_{d}\right)_{n+1}^{(k)}
$$

\section{Algorithm performance and example applications}

The numerical procedures outlined in Sects. 4 and 5 for the elastoplastic model with generalized hardening developed to describe mechanical and environmental loading effects on weak calcarenite rocks have been implemented into the research oriented FE platform GEHOMADRID [19], which incorporates fully coupled threephase consolidation and transport of chemical species in the pore water, see [18].

In the following, the results of some representative numerical simulations carried out at the single element level and at the Initial-Boundary Value Problem (IBVP) level - are presented in order to investigate the accuracy and efficiency characteristic of the proposed stress-point algorithm, and to demonstrate the predictive capability of the model in reproducing the observed behavior of a typical Apulian calcarenite rock under complex loading conditions in specially designed laboratory experiments.

In the numerical simulations reported in the following, two different sets of material constants have been adopted, as shown in Tab. 3. The role played by each material constant and the procedures for their experimental determination have already been discussed in refs. $[11,10]$ and will not be repeated here.

\subsection{Convergence and accuracy properties of the GBE algorithm}

In a first series of numerical tests, the performance of the GBE algorithm has been tested by simulating a series of standard laboratory tests - including onedimensional compression and axisymmetric drained compression tests - performed with varying step sizes, in order to assess the convergence rate of the numerical solution. In the second series of tests, the accuracy properties of the GBE algorithm have been investigated more extensively by producing isoerror maps [40], including both mechanical and environmental loading conditions. 
Table 3 Material constants adopted in the numerical simulations.

\begin{tabular}{|c|cccccccc|}
\hline $\begin{array}{c}\text { constant } \\
\text { set \# }\end{array}$ & $\begin{array}{c}\alpha \\
(-)\end{array}$ & $\begin{array}{c}\hat{\kappa} \\
(-)\end{array}$ & $\begin{array}{c}G_{0} \\
(\mathrm{kPa})\end{array}$ & $\begin{array}{c}p_{r} \\
(\mathrm{kPa})\end{array}$ & $\begin{array}{c}\rho_{s} \\
(-)\end{array}$ & $\begin{array}{c}\xi_{s} \\
(-)\end{array}$ & $\begin{array}{c}\rho_{t} \\
(-)\end{array}$ & $\begin{array}{c}\xi_{t} \\
(-)\end{array}$ \\
\hline 1 & 0.0 & 67 & 18000 & $1.0 \cdot 10^{6}$ & 16.66 & -0.1 & 15.0 & 1.0 \\
2 & 0.0 & 73 & 16500 & $1.0 \cdot 10^{6}$ & 16.66 & -0.1 & 15.0 & 1.0 \\
\hline \hline constant & $a_{g}$ & $m_{g}$ & $M_{g c}$ & $M_{g e}$ & $a_{f}$ & $m_{f}$ & $M_{f c}$ & $M_{f e}$ \\
set \# & $(-)$ & $(-)$ & $(-)$ & $(-)$ & $(-)$ & $(-)$ & $(-)$ & $(-)$ \\
\hline 1 & $1.0 \cdot 10^{6}$ & 1.2 & 1.675 & 1.292 & 0.75 & 1.5 & 1.3 & 1.0 \\
2 & $1.0 \cdot 10^{6}$ & 1.2 & 1.675 & 1.292 & 0.75 & 1.5 & 1.3 & 1.0 \\
\hline \hline constant & $\delta_{b}$ & $\xi_{d, \mathrm{cr}}$ & $S_{r, \mathrm{cr}}$ & $R_{c}$ & $R_{E}$ & $k$ & $\Delta_{d}$ & $C_{\max }$ \\
set \# & $(-)$ & $(-)$ & $(-)$ & $(-)$ & $(-)$ & $(-)$ & $(-)$ & $(-)$ \\
\hline 1 & 4 & 0.4 & 0.3 & 0.72 & 0.48 & 5.0 & 0.05 & 0.33 \\
2 & 4 & 0.3 & 0.3 & 0.72 & 0.48 & 5.0 & 0.20 & 0.33 \\
\hline
\end{tabular}

Table 4 Single-element testing program.

\begin{tabular}{|c|ccccccc|}
\hline $\begin{array}{c}\text { Run } \\
\#\end{array}$ & $\begin{array}{c}\text { loading } \\
\text { path }\end{array}$ & $\begin{array}{c}\text { constant } \\
\text { set \# }\end{array}$ & $\begin{array}{c}p_{0} \\
(\mathrm{kPa})\end{array}$ & $\begin{array}{c}q_{0} \\
(\mathrm{kPa})\end{array}$ & $\begin{array}{c}p_{s 0} \\
(\mathrm{kPa})\end{array}$ & $\begin{array}{c}p_{t 0} \\
(\mathrm{kPa})\end{array}$ & $\begin{array}{c}\# \\
\text { of steps }\end{array}$ \\
\hline \hline 1 & OED & 1 & 0.0 & 0.0 & 1200.0 & 320.0 & 500 \\
2 & OED & 1 & 0.0 & 0.0 & 1200.0 & 320.0 & 100 \\
3 & OED & 1 & 0.0 & 0.0 & 1200.0 & 320.0 & 50 \\
4 & OED & 1 & 0.0 & 0.0 & 1200.0 & 320.0 & 10 \\
\hline 5 & TX-CD & 1 & 2000.0 & 0.0 & 1000.0 & 380.0 & 500 \\
6 & TX-CD & 1 & 2000.0 & 0.0 & 1000.0 & 380.0 & 100 \\
7 & TX-CD & 1 & 2000.0 & 0.0 & 1000.0 & 380.0 & 50 \\
8 & TX-CD & 1 & 2000.0 & 0.0 & 1000.0 & 380.0 & 25 \\
\hline
\end{tabular}

Note: all tests performed assuming fully saturated conditions $\left(S_{r}=1\right)$ and $\xi_{d 0}=0$.

In both series of simulations, a global relative error tolerance TOL $=1.0 \times 10^{-9}$ has been adopted. In order to quantify the accuracy of the numerical solutions obtained, the following, non-dimensional relative error measure has been adopted:

$$
\mathrm{ERR}:=\frac{\sqrt{\left(\boldsymbol{\sigma}-\boldsymbol{\sigma}^{*}\right) \cdot\left(\boldsymbol{\sigma}-\boldsymbol{\sigma}^{*}\right)+\left(\boldsymbol{q}-\boldsymbol{q}^{*}\right) \cdot\left(\boldsymbol{q}-\boldsymbol{q}^{*}\right)}}{\sqrt{\left(\boldsymbol{\sigma}^{*} \cdot \boldsymbol{\sigma}^{*}\right)+\left(\boldsymbol{q}^{*} \cdot \boldsymbol{q}^{*}\right)}}
$$

where $\left(\boldsymbol{\sigma}^{*}, \boldsymbol{q}^{*}\right)$ represents the "exact" reference solution for each loading condition considered. As closed form solutions for the differential-algebraic problem governed by eqs. (52) - (56) with prescribed initial data are not generally available, in the present work the reference solutions for each of the test considered have been obtained by integrating the evolution equation numerically, using the BFD algorithm a very large number of steps $($ NSTEP $=10000)$.

\subsubsection{Simulation of single element tests}

The complete program of numerical simulation of single element tests performed for the assesment of the convergence properties of the GBE algorithm is detailed in Tab. 4. In the table, information on the initial state of the material assumed in each simulation are also provided. 


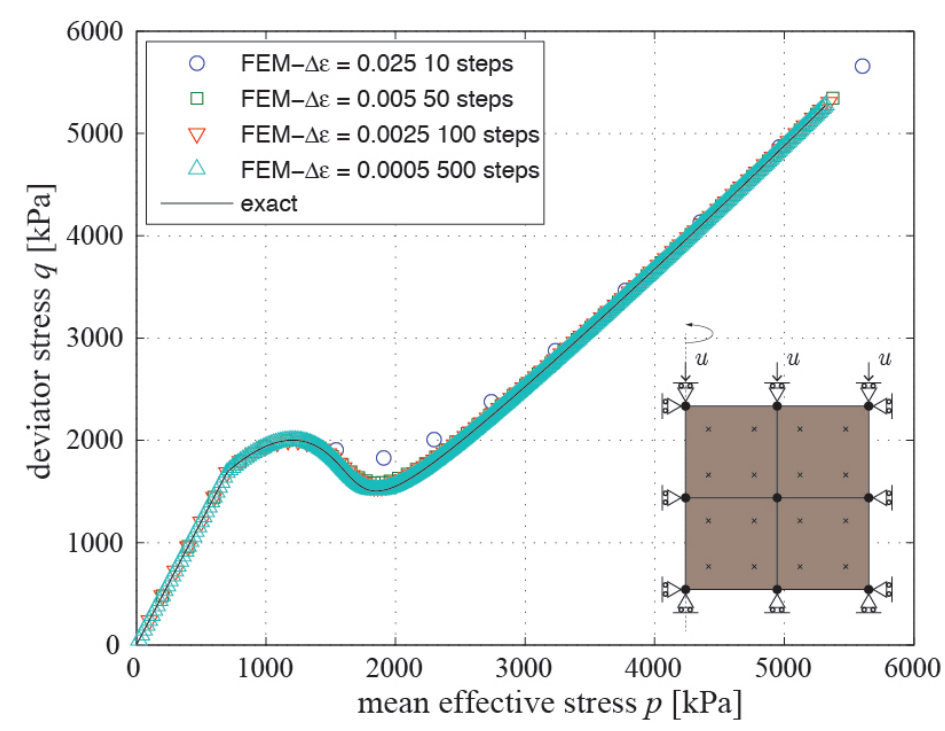

Fig. 4 Numerical simulations of a OED compression tests: computed stress paths.

Tests \#1 to 4 refer to the simulation of a one-dimensional (OED) compression test of a saturated calcarenite rock, starting from an initial stress-free state. In the different simulations, a total axial strain increment $\Delta \epsilon_{a}=0.25$ is applied in 10 , 50,100 and 500 steps, respectively. It is worth noting that, as the response of the material to the imposed deformations is characterized by a very large variation of the mean effective stress - and thus by a strong non-linearity - the OED loading represents a rather challenging benchmark for the GBE algorithm.

The results obtained are compared with the "exact" solution in Fig. 4, which shows the predicted stress paths in the $q: p$ plane. In all the simulations, the initial part of the stress path is elastic, with a stress ratio controlled by the material Poisson's ratio. As soon as the initial yield surface is reached, the stress path bends to the right, with a continuous decrease of the stress ratio until a final constant value, for large axial strains, corresponding to the constant $K_{0}$ coefficient of the virgin, unboded material. As soon as plastic deformations start developing, mechanical debonding takes place, producing a progressive reduction of $p_{t}$ and $p_{m}$ until they reduce to zero towards the end of the test.

The comparison between the different numerical solutions show a remarkable convergence towards the reference one as the number of steps increase. As a matter of fact, the 100 and 200 step solutions appear practically indistinguishable from the "exact" stress-path, and even the 10 step solution lies not too far from the reference one.

Tests \#5 to 8 refer to the simulation of an axisymmetric drained compression (TX-CD) compression test of a saturated calcarenite rock, starting from an initial isotropic state state with $p_{0}=2000 \mathrm{kPa}$. In the different simulations, a very large total axial strain increment $\Delta \epsilon_{a}=0.625$ is applied, at constant radial effective 


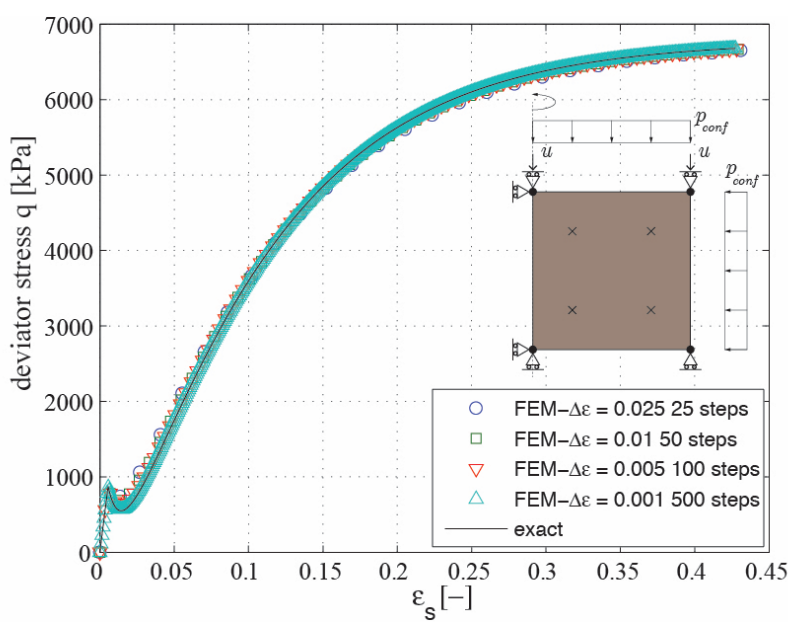

(a)

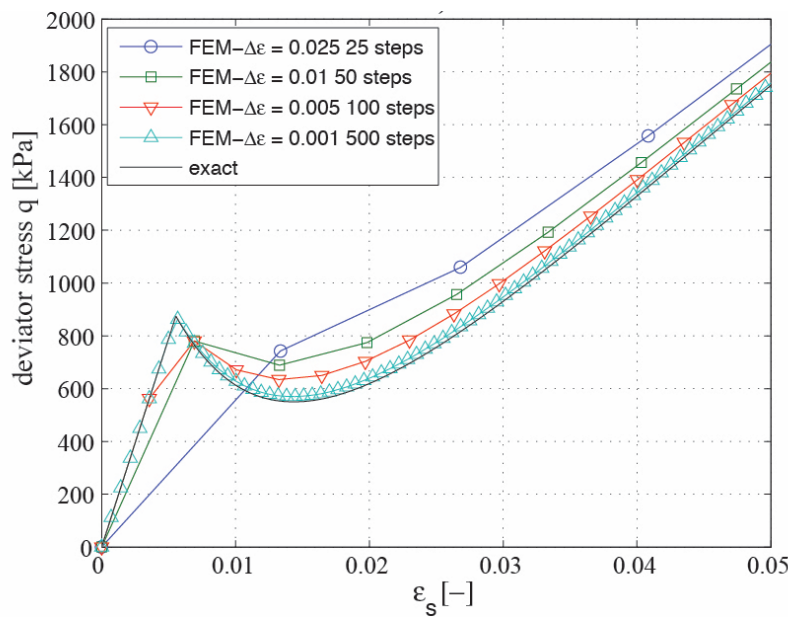

(b)

Fig. 5 Numerical simulations of a TX-CD compression tests: a) stress-strain curves in the $q: \epsilon_{s}$ plane; b) magnification of the initial portion of the stress-strain curves.

The results obtained are compared with the reference solution in Fig. 5, which shows the predicted stress-strain response in the $q: \epsilon_{s}$ plane. Except for the simulations with 25 and 50 steps, characterized by trial stress increments so large that plastic loading occurs right from the first step, the initial part of the stress-strain curve shows an elastic response, followed by an initial softening branch. This is due to the strong reduction in the yield locus size associated with mechanical debonding, which - in the initial stage of the test - prevails on the density hardening mechanism. As plastic deformations increase, the bonding-related internal variables $p_{t}$ and $p_{m}$ go to zero and $p_{s}$ remains the only active hardening variables up to ultimate failure. 


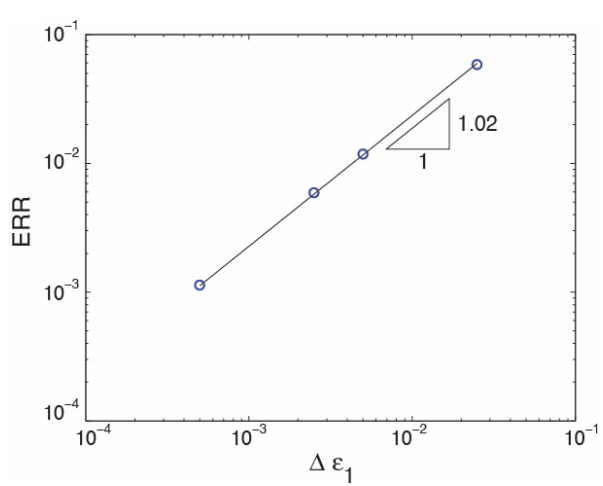

(a)

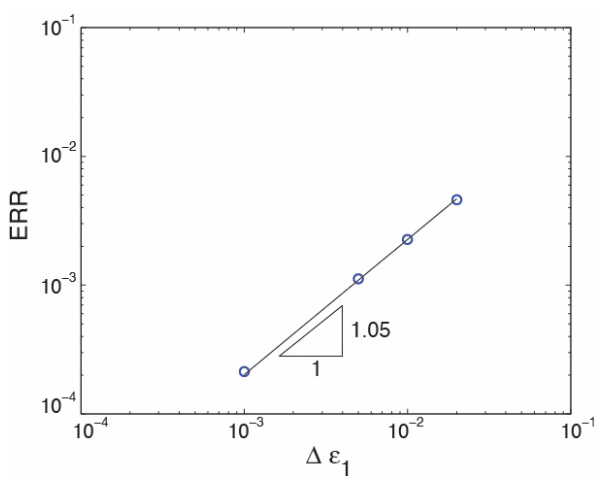

(b)

Fig. 6 Convergence analysis: a) OED compression test; b) TX-CD compression test.

As in the OED test simulations, the comparison between the different numerical solutions show a very good convergence towards the reference one as the number of steps increase, with a fairly accurate prediction already at 100 steps $\left(\Delta \epsilon_{a}=\right.$ $6.25 \times 10^{-3}$ for each step).

A more complete picture of the convergence properties of the GBE algorithm for the two different loading conditions is provided by Fig. 6, which provides the evolution of the integration error ERR with the number of steps for the two series of simulations of Tab. 4. A least squares power fitting of the data points in the two figures provides a convergence rate of 1.02 and 1.05, respectively, in line with the expected performance of a first-order accurate algorithm.

\subsubsection{Isoerror maps}

A sistematic investigation of the accuracy properties of the GBE algorithm for a wide class of mechanical and environmental loading conditions can be performed using the concept of isoerror map [40]. A isoerror map provides in graphical form the integration error associated to the numerical solution provided by the GBE algorithm in a single-step calculation as a function of the "load" increment size.

In our case, as the evolution of the state of the material is driven by changes in strain and environmental process variables, the load increments are represented by the principal strain increments $\Delta \epsilon_{A}(A=1,2$ or 3$)$, the change in the degree of saturation $\Delta S_{r}$ and the change in mass dissolution ratio $\Delta \xi_{d}$. In order to construct two-dimensional representations, the attention has been focused on plane strain processes $\left(\Delta \epsilon_{3}=0\right)$.

In particular, the following cases have been considered:

A) fully dry $\left(S_{r}=0\right)$ or saturated $\left(S_{r}=1\right)$ intact material $\left(\xi_{d 0}=0\right)$, with no chemical degradation $\left(\Delta \xi_{d}=0\right)$;

B) fully dry intact material $\left(S_{r 0}=0, \xi_{d 0}=0\right)$ subject to four different levels of $\operatorname{STD}\left(\Delta S_{r}=0.1,0.2,0.3\right.$ and 1.0$)$, with no chemical degradation $\left(\Delta \xi_{d}=0\right)$;

C) fully saturated intact material $\left(S_{r 0}=1, \xi_{d 0}=0\right)$ subject to four levels of LTD $\left(\Delta \xi_{d}=0.1,0.2,0.3\right.$ and 0.35$)$; 
Table 5 Isoerror map testing program.

\begin{tabular}{|c|cccccccc|}
\hline $\begin{array}{c}\text { map } \\
\#\end{array}$ & $\begin{array}{c}\sigma_{10} \\
(\mathrm{kPa})\end{array}$ & $\begin{array}{c}\sigma_{20} \\
(\mathrm{kPa})\end{array}$ & $\begin{array}{c}\sigma_{30} \\
(\mathrm{kPa})\end{array}$ & $\begin{array}{c}S_{r 0} \\
(-)\end{array}$ & $\begin{array}{c}\Delta \epsilon_{1, \max } \\
(\%)\end{array}$ & $\begin{array}{c}\Delta \epsilon_{2, \max } \\
(\%)\end{array}$ & $\begin{array}{c}\Delta S_{r} \\
(-)\end{array}$ & $\begin{array}{c}\Delta \xi_{d} \\
(-)\end{array}$ \\
\hline \hline A1 & 2511 & 189 & 189 & 0.0 & 2.03 & 2.03 & 0.0 & 0.0 \\
A2 & 2511 & 189 & 189 & 1.0 & 2.03 & 2.03 & 0.0 & 0.0 \\
\hline B1 & 2511 & 189 & 189 & 0.0 & 2.03 & 2.03 & 0.1 & 0.0 \\
B2 & 2511 & 189 & 189 & 0.0 & 2.03 & 2.03 & 0.2 & 0.0 \\
B3 & 2511 & 189 & 189 & 0.0 & 2.03 & 2.03 & 0.3 & 0.0 \\
B4 & 2511 & 189 & 189 & 0.0 & 2.03 & 2.03 & 1.0 & 0.0 \\
\hline C1 & 1739 & 131 & 131 & 1.0 & 3.17 & 3.17 & 0.0 & 0.1 \\
C2 & 1739 & 131 & 131 & 1.0 & 3.17 & 3.17 & 0.0 & 0.2 \\
C3 & 1739 & 131 & 131 & 1.0 & 3.17 & 3.17 & 0.0 & 0.3 \\
C4 & 1739 & 131 & 131 & 1.0 & 3.17 & 3.17 & 0.0 & 0.35 \\
\hline D1 & 1739 & 131 & 131 & 1.0 & 3.17 & 1.9 & 0.0 & 0.350 \\
D2 & 1739 & 131 & 131 & 1.0 & 3.17 & 1.9 & 0.0 & 0.354 \\
D3 & 1739 & 131 & 131 & 1.0 & 3.17 & 1.9 & 0.0 & 0.358 \\
D4 & 1739 & 131 & 131 & 1.0 & 3.17 & 1.9 & 0.0 & 0.360 \\
\hline
\end{tabular}

Note: all tests performed with set \#1 of material constants of Tab. 3, starting from an initially intact state $\left(\xi_{d 0}=0\right)$.

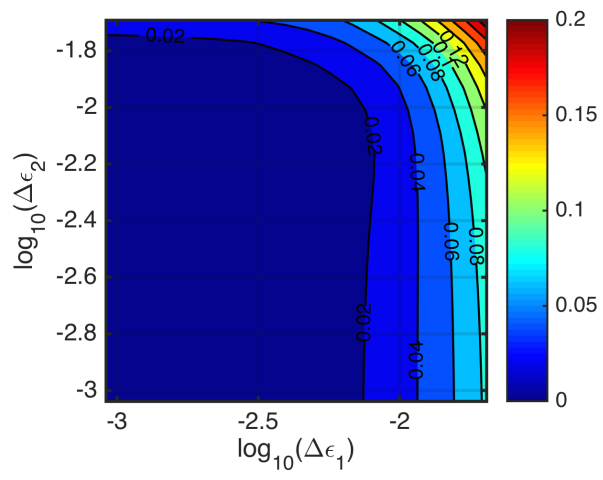

(a)

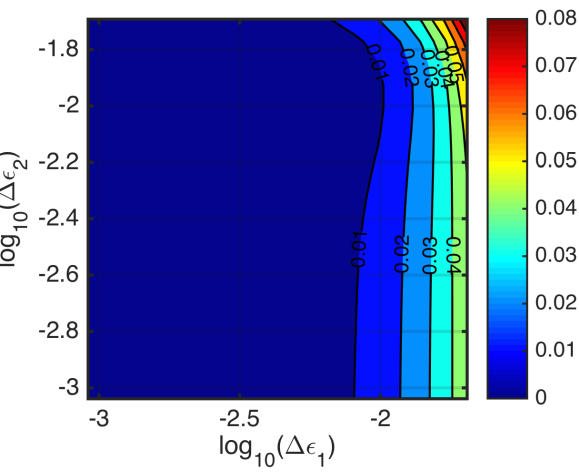

(b)

Fig. 7 Isoerror maps for simulation group A: a) dry material; b) fully saturated material.

D) fully saturated intact material $\left(S_{r 0}=1, \xi_{d 0}=0\right)$ subject to four levels of LTD including grain dissolution $\left(\Delta \xi_{d}=0.35,0.354,0.358\right.$ and 0.36$)$.

The full program of numerical simulations, including the initial state of the material for each group of tests is detailed in Tab. 5

The isoerror maps corresponding to simulation groups (A), (B), (C) and (D) are shown in Figs. 7 to 10, respectively. In each map, the integration error ERR is plotted as a function of the principal strain increments $\Delta \epsilon_{1}$ and $\Delta \epsilon_{2}$ for given values of $\Delta S_{r}$ and $\Delta \xi_{d}$.

From all the maps, it can be observed that the integration error remains very small for a relatively large region of the loading space. As far as purely mechanical load increments are concerned (Fig. 7), it can be observed that significantly larger errors are obtained in case (A1) than in case (A2) for the same level of applied strain increment, since the dry material is stiffer than the saturated material. The 


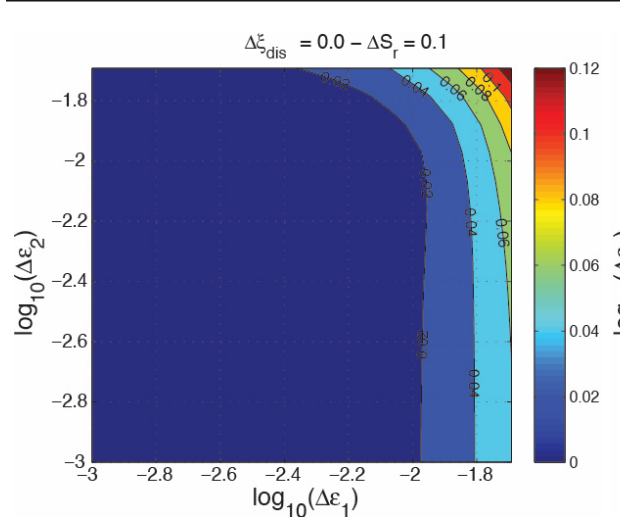

(a)

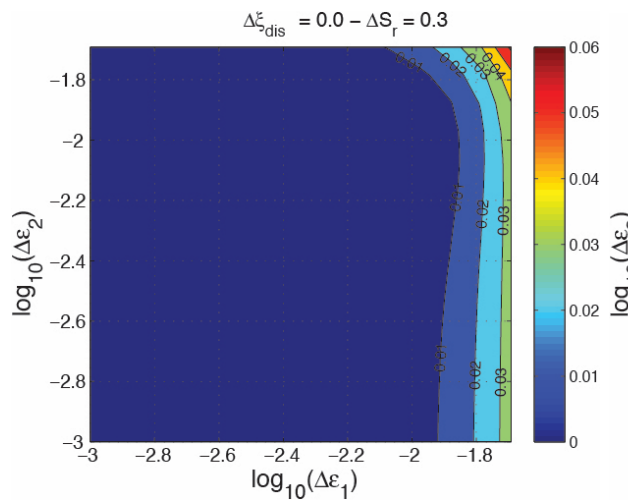

(c)

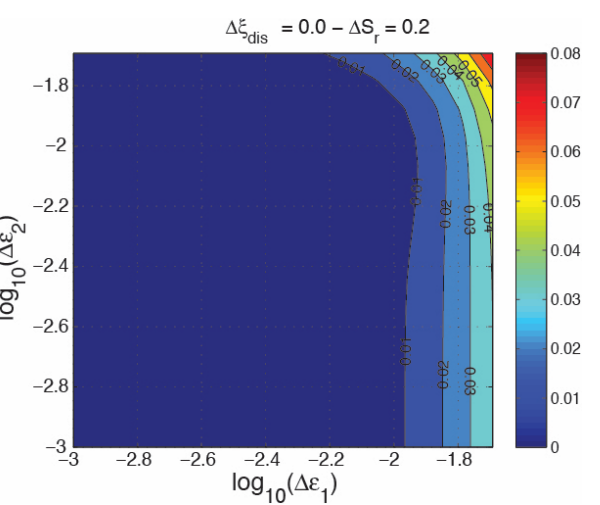

(b)

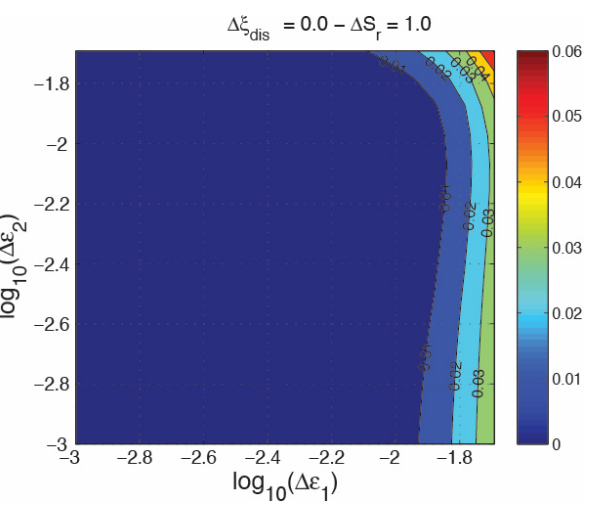

(d)

Fig. 8 Isoerror maps for simulation group B: a) $\Delta S_{r}=0.1$; b) $\Delta S_{r}=0.2$; c) $\Delta S_{r}=0.3$; d) $\Delta S_{r}=1.0$.

results obtained for hydro-mechanical loading processes (cases B1-B4, Fig. 8) confirm this finding. As $\Delta S_{r}$ increases up to 0.3 , the error associated to a given strain increment reduces significantly. No significant differences in computed errors are observed for further saturation increments up to $S_{r}=1.0$. Interestingly, a similar effect can be observed in chemo-mechanical loading processes not inducing grain dissolution (cases C1-C4, Fig. 9), in which the error computed at a given strain increment also reduces slightly as $\Delta \xi_{d}$ increases, even if the degree of nonlinearity induced by the LTD process is stronger than the one associated to the STD one, see Fig. 2.

In chemo-mechanical loading processes where $\Delta \xi_{d}$ is sufficiently high to induce chemical deformations (cases D1-D4, Fig. 10), the pattern of error distribution changes completely. In fact, small total strain increments associated to large increments of $\Delta \xi_{d}$ give rise to large variation in elastic strain increments and thus to large stress changes. This is why, in this case, the computed error tends to increase towards the origin of the total strain increment space, with errors increasing with $\Delta \xi_{d}$ at any given strain increment. 


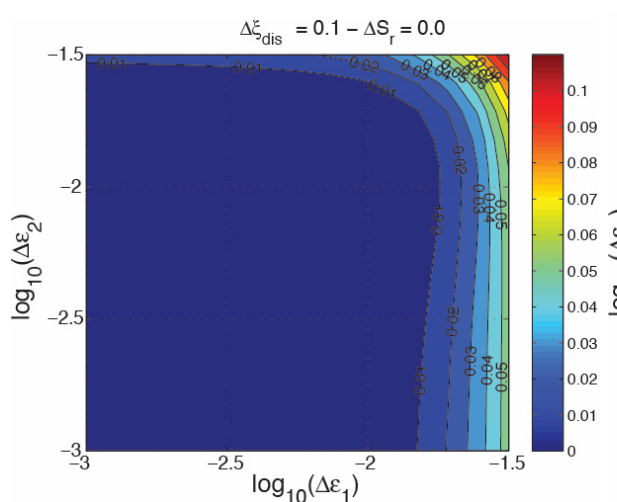

(a)

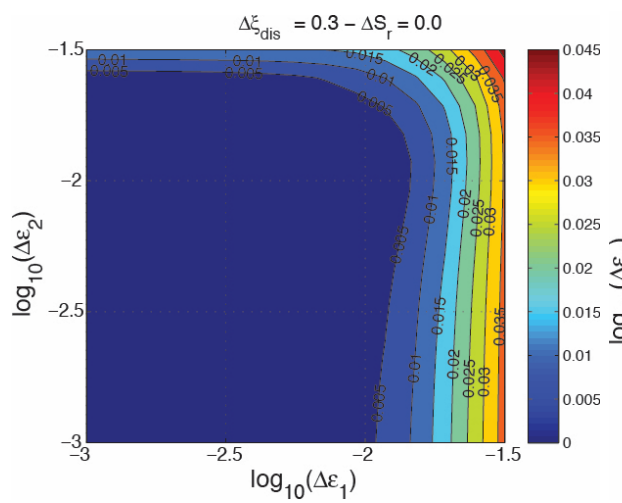

(c)

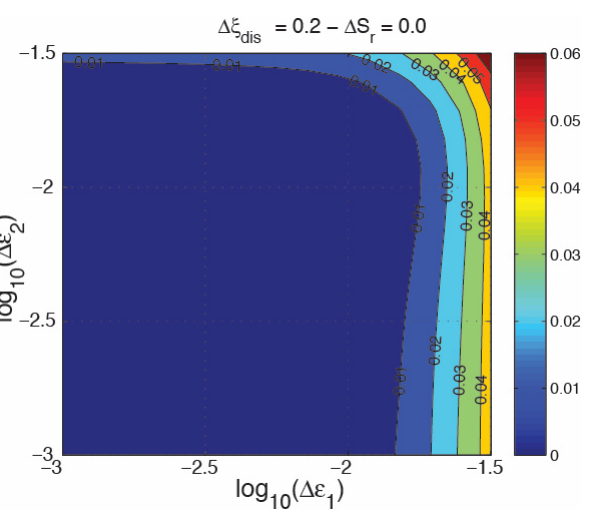

(b)

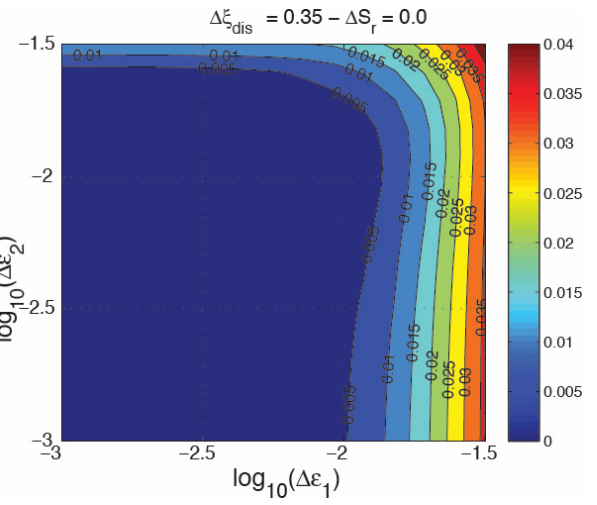

(d)

Fig. 9 Isoerror maps for simulation group C: a) $\Delta \xi_{d}=0.1$; b) $\Delta \xi_{d}=0.2$; c) $\Delta \xi_{d}=0.3$; d) $\Delta \xi_{d}=0.35$.

\subsection{Application to representative IBVPs}

${ }_{680}$ In order to demonstrate the predictive capabilities of the proposed elastoplastic model for calcarenites presented in Sect. 3 and the applicability of the numerical procedures outlined in Sects. 4 and 5 to IBVPs of practical interest, two additional numerical simulations are presented: the modeling of a chemo-hydro-mechanical one-dimensional (OED) compression test performed by [9] on a cylindrical specimen of Gravina calcarenite, and the simulation of the ground subsidence induced by chemical degradation around underground man-made cavities in calcarenite rocks.

\subsubsection{Chemo-hydro-mechanical one-dimensional compression test}

A picture of the experimental apparatus used to perform the test - designed by

[6] - and of the FE model adopted in its simulation are shown in Fig. 11(a) and 11(b), respectively.

The laboratory test has been performed in the following 5 stages: 


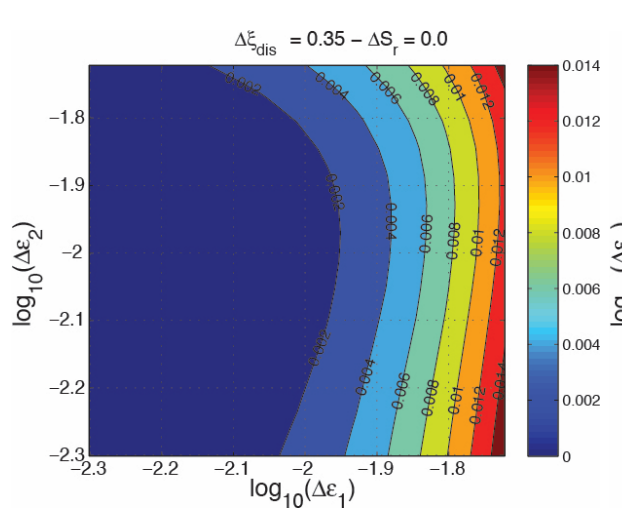

(a)

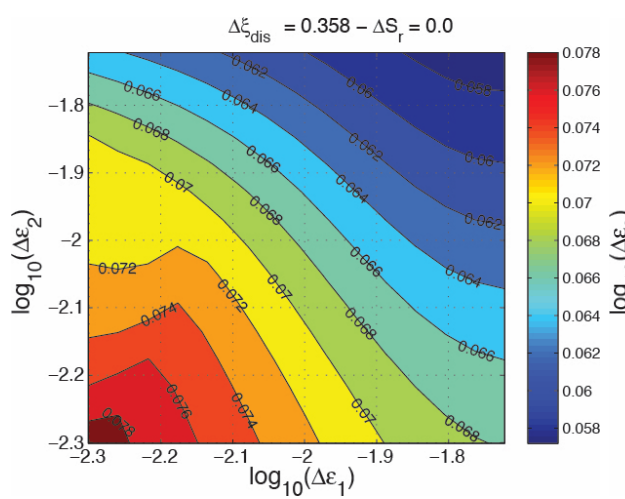

(c)

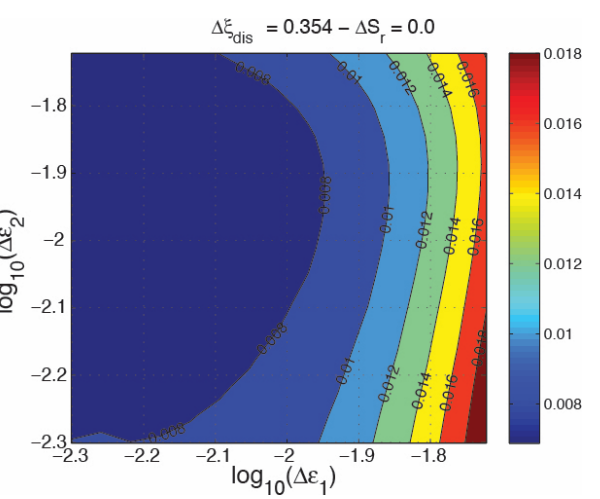

(b)

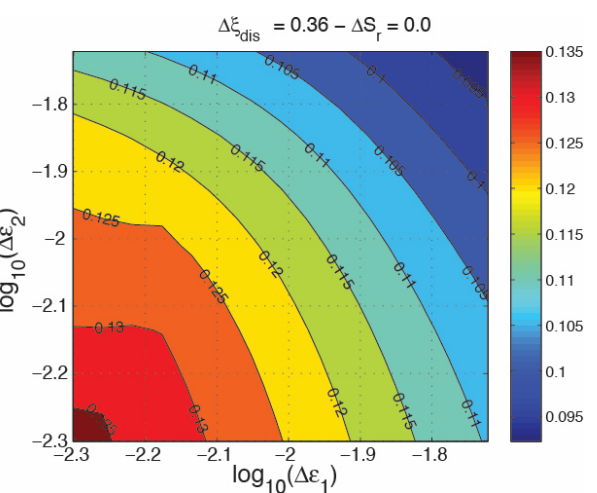

(d)

Fig. 10 Isoerror map for simulation group D, incorporating grain dissolution: a) $\Delta \xi_{d}=0.3$; b) $\Delta \xi_{d}=0.354$; c) $\Delta \xi_{d}=0.358$; ) $\Delta \xi_{d}=0.36$.

1. mechanical loading stage $\mathrm{O}-\mathrm{A}$ : the dry and intact specimen $\left(S_{r 0}=0, \xi_{d 0}=0\right)$ is compressed under one-dimensional conditions until a total axial stress $\tau_{a}=$ $2005 \mathrm{kPa}$ is reached;

2. dry creep stage A-B: the specimen is allowed to deform freely at constant $\tau_{a}$ $=2005 \mathrm{kPa}$ for about $20 \mathrm{~min}$.;

3. wetting stage $\mathrm{B}-\mathrm{C}$ : under the same constant vertical stress, the dry specimen is progressively inundated by flushing water from the bottom boundary at a constant imposed water flux, attaining full saturation of the sample in about 2 min.;

4. saturated creep stage $\mathrm{C}-\mathrm{D}$ : the specimen is allowed to deform freely at constant $\tau_{a}=2005 \mathrm{kPa}$ for about $10 \mathrm{~min}$;

5. chemical degradation stage $\mathrm{D}-\mathrm{E}$ : an acid solution at $\mathrm{pH}=2.5$ is flushed from the bottom boundary at a constant flow rate $Q=1.7 \mathrm{l} / \mathrm{h}$, at constant $\tau_{a}$, for $220 \mathrm{~min}$.

The reader is referred to [9] for further details on the experimental procedure. Being the model rate-independent, in the FE modeling of the test only stages (1), (3) and (5) have been simulated. The simulation has been performed with the 


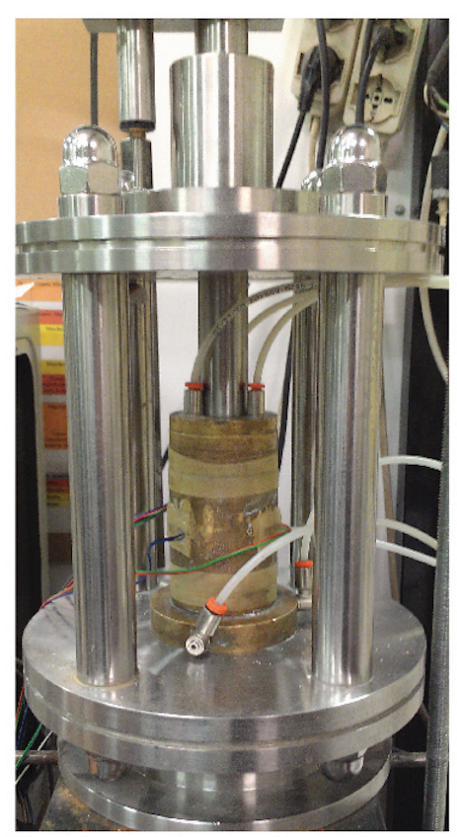

(a)

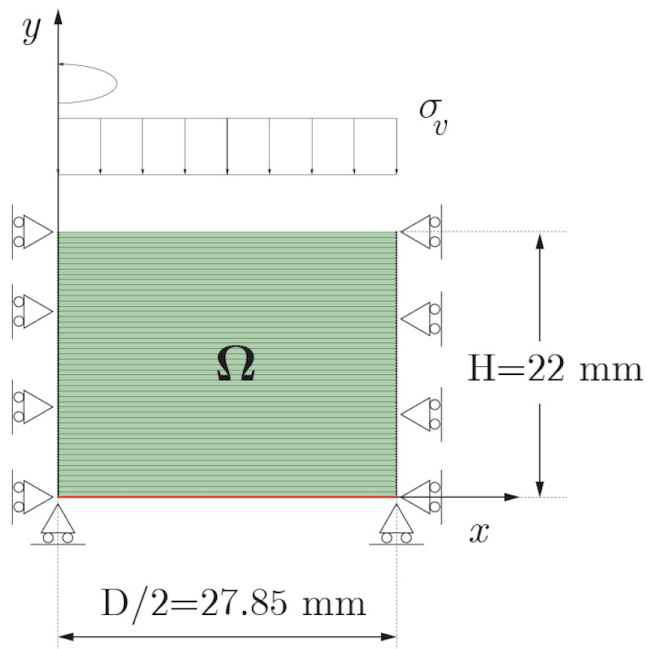

(b)

Fig. 11 Chemo-hydro-mechanical one-dimensional compression test [9]: a) experimental apparatus; b) geometry and FE discretization of the numerical model.

material constant set \#2 of Tab. 3. The FE discretization adopted is composed of 128 4-noded axisymmetric bilinear interpolation elements, placed on a single column due to the one-dimensional character of the flow and deformation processes. The relatively large number of elements adopted allows to capture well the strong hydraulic and chemical gradients occurring within the specimen during stages (3) 5 and (5).

In order to model the hydraulic conduction process, the saturated permeability of the material has been set to $1.0 \times 10^{-5} \mathrm{~m} / \mathrm{s}$; a very simple linear water retention curve fitted on the experimental data provided by [9] has been adopted in the unsaturated regime.

As in [18], the chemical transport processes associated with the chemical dissolution of calcite by an acid solution occurring in stage (5) have been modeled using a two-stage operator splitting approach, which incorporates a Taylor-Galërkin scheme for the advective transport and a Runge-Kutta (4th order) scheme for the treatment of the source term. In the GeHoMADRID FE implementation, the hydro-mechanical problem (HM) and the reactive transport problem $(\mathrm{C})$ are coupled by means of a staggered approach, see [18] for further details.

The initial concentration of calcite in the specimen is evaluated from the initial mass of the intact rock $(70.23 \mathrm{~g})$ divided by the pore volume, thus obtaining $\left[\mathrm{CaCO}_{3(\mathrm{~s})}\right]=2.52 \mathrm{~g} / \mathrm{cm}^{3}\left(=25200 \mathrm{~mol} / \mathrm{m}^{3}\right)$. The acid flux is imposed by prescribing a fixed $\left[\mathrm{H}^{+}\right]=3.162 \mathrm{~mol} / \mathrm{m}^{3}$ at the bottom base of the specimen and a constant upward flow velocity $v_{y}=1.83 \times 10^{-4} \mathrm{~m} / \mathrm{s}$ in the entire domain (corre- 


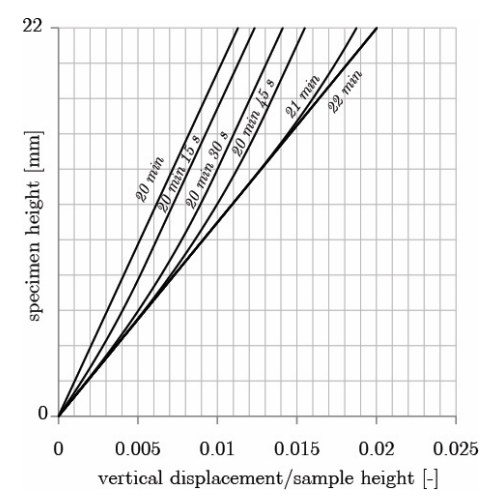

(a)

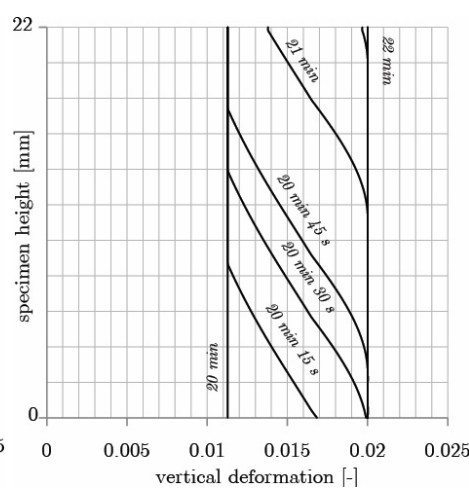

(b)

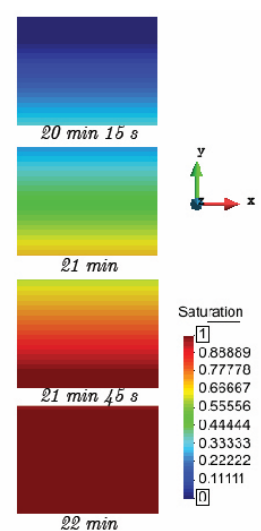

(c)

Fig. 12 Chemo-hydro-mecanical OED compression test. Results from wetting stage (3): a) isochrones of normalized axial displacement; b) isochrones of axial deformation; c) contour maps of degree of saturation $S_{r}$ at four different time stations.

sponding to a constant $Q=1.7 \mathrm{l} / \mathrm{h})$. The mass balance constants appearing in the $\left[\mathrm{H}^{+}\right]$and $\left[\mathrm{CaCO}_{3(\mathrm{~s})}\right]$ transport equations have been set to $K_{1}=-2 \times 10^{-6}$ and $K_{2}=1 \times 10^{-5}$, respectively.

During stage loading (1), the size of the elastic domain is sufficiently large that the final state of the material is elastic. Some selected results from the FE simulation of wetting stage (3) and chemical degradation stage (5) are shown in Figs. 12 to 15 .

The evolution with time of the axial displacement and deformation profiles, as well as four different snapshots of the degree of saturation distribution within the specimen during stage (3) are shown in Fig. 12. The final, homogeneous state of the specimen at full saturation $(t=22 \mathrm{~min}$.) is still elastic. During the wetting process, the constitutive axial stress $\sigma_{a}$ first increases and then reduces slightly - due to the relatively large porosity of the material, the suctions developed inside the specimen are quite low. Hovever, the specimen undergoes a monotonic compaction process, initiating at the bottom and progressing upward with the saturation front. This is the consequence of the reduction in the solid skeleton stiffness associated with the STD process.

Fig. 13 provides the time evolution of the concentration of $\mathrm{H}^{+}$ions in the pore water, as well as of water $\mathrm{pH}$ at the bottom (in) and top (out) bases of the specimen. This last data is compared with experimental measurements in Fig. 13(a). The corresponding evolution in space and time of the mass dissolution ratio $\xi_{d}$ is given in Fig. 14, in terms of average value vs. time - Fig. 14(a) - and isochrones of $\xi_{d}$ at different time stations - Fig. 14(b). Fig. 14(a) also provides the evolution in time of $\xi_{d}$ which would be obtained if $\mathrm{pH}$ was uniform throughout the specimen and equal to the $\mathrm{pH}$ at the inflow and outflow boundaries, respectively. The comparison with the experimentally measured mass dissolution ratio at the end of the test corresponds quite well with the predicted average value, while the "homogeneous" (single element) computations either underpredict (outflow $\mathrm{pH}$ ) or overpredict (inflow $\mathrm{pH}$ ) the experimental data. This last observation shows that - 


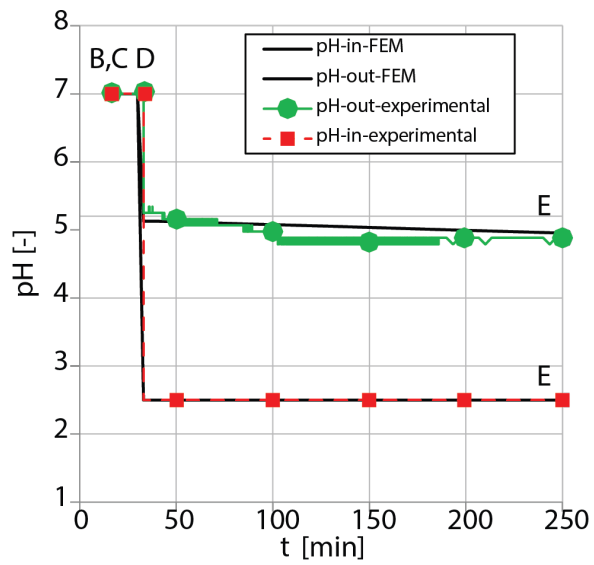

(a)

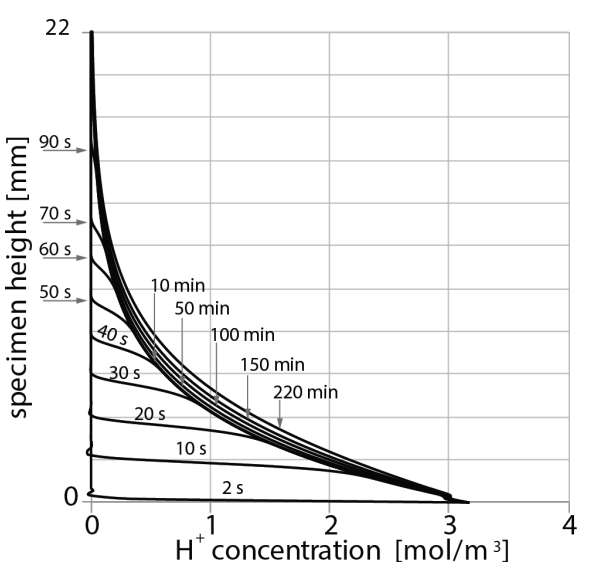

(b)

Fig. 13 Chemo-hydro-mecanical OED compression test. Results from chemical degradation stage (5): a) time evolution of pore water $\mathrm{pH}$ at the bottom (in) and top (out) bases of the specimen; b) isochrones of $\left[\mathrm{H}^{+}\right]$(time labels refer to the beginning of the chemical degradation stage).

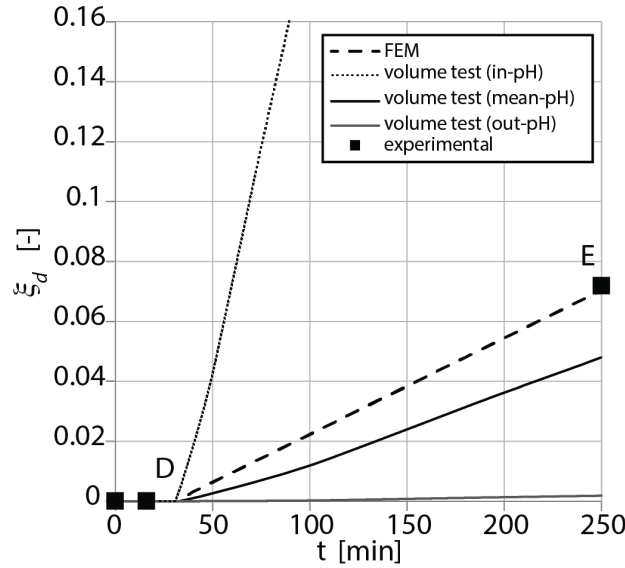

(a)

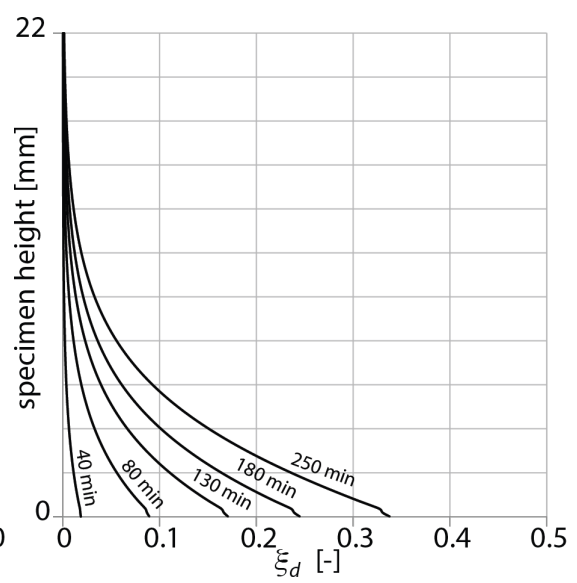

(b)

Fig. 14 Chemo-hydro-mecanical OED compression test. Results from chemical degradation stage (5): a) time evolution of mass dissolution ratio $\xi_{d} ; \mathrm{b}$ ) isochrones of $\xi_{d}$.

no matter how simple the experimental setup - the chemo-hydro-mecanical OED compression test must be interpreted as an IBVP, rather than as a homogeneous test, when used for model calibration.

The progressive increase of $\xi_{d}$ with time inside the specimen induces a LTD process which causes a progressive reduction in size of the yield locus until the current stress state touches the yield surface. Any further reduction of $p_{t}$ and $p_{m}$ is accompanied by the development of contractant plastic strains which, inducing a compensation hardening effect, produce an increment in $p_{s}$ and an increase in the radial stress state sufficient to maintain the current state on the yield sur- 


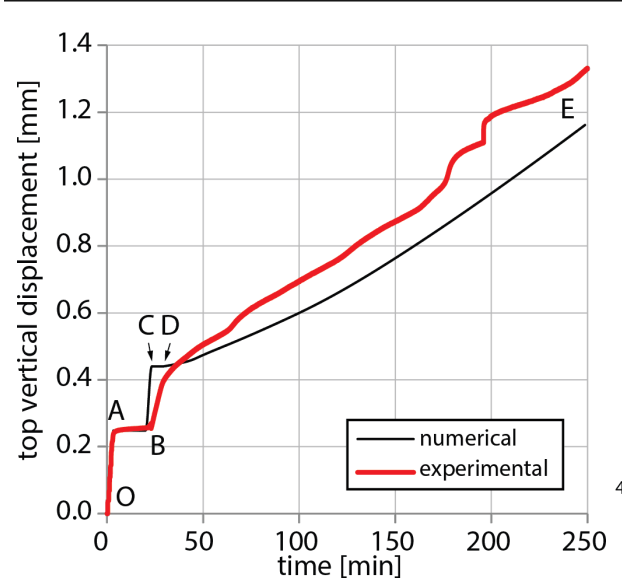

(a)

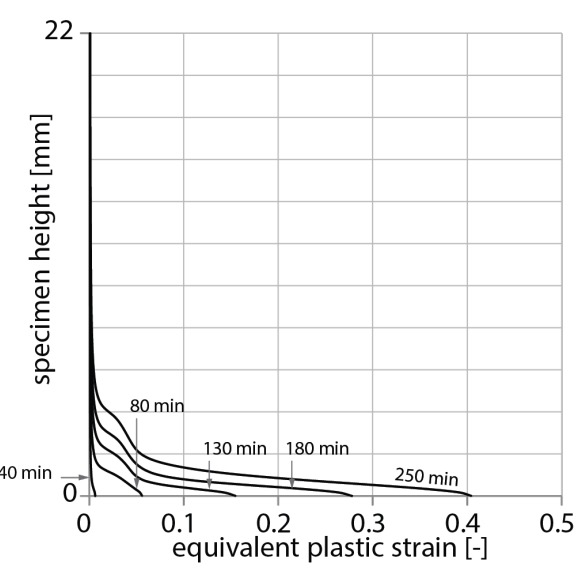

(b)

Fig. 15 Chemo-hydro-mecanical OED compression test. Results from chemical degradation stage (5): a) time evolution of axial displacement at specimen top base; b) isochrones of equivalent plastic strain $\epsilon_{\mathrm{eq}}^{p}$.

face, without violating the consistency condition $(f=0 ; \dot{f}=0)$. For $\xi_{d}>\xi_{d, \mathrm{cr}}$, contractant chemical deformations due to grain dissolution add up to the plastic deformations. As both plastic and chemical deformations are characterized by a contractant volumetric component, they induce a time-dependent downward axial displacement of the top loading plate, which can be measured with a standard displacement transducer.

Fig. 15(a) shows a comparison between measured and predicted evolution of the top platen displacement with time, together with the isochrones of the equivalent plastic strain $\epsilon_{\mathrm{eq}}^{p}=\epsilon_{s}^{p}$. The match between predicted and computed displacement histories during stage (5) of the test appears very good. It is worth noting that:

i) at each time station, the top platen displacement is the result of the spatial integration of a non-uniform axial deformation profile along the specimen axis. Therefore, the interpretation of the test results in terms of homogeneous response of the material is not possible, as already observed previously;

ii) the increase of top platen displacement with time at almost constant speed up to the end of the test is a consequence of chemical deformations, which continue to develop even after the material has been fully debonded $\left(p_{t}=p_{m}\right.$ $=0)$. This effect is well captured by the model.

In addition, the results in Fig. 15(b) indicate that the chemo-mechanical damage tends to concentrate in the bottom part of the specimen, where the mass dissolution ratio attains its largest values.

\subsubsection{Ground subsidence above underground excavations in calcarenite rocks}

The second IBVP considered concerns the evaluation of ground subsidence above underground excavations in calcarenite rocks undergoing chemical degradation from the tunnel surface due to the exposition to aggressive atmospheric environment within the cavities, see e.g. [34]. 


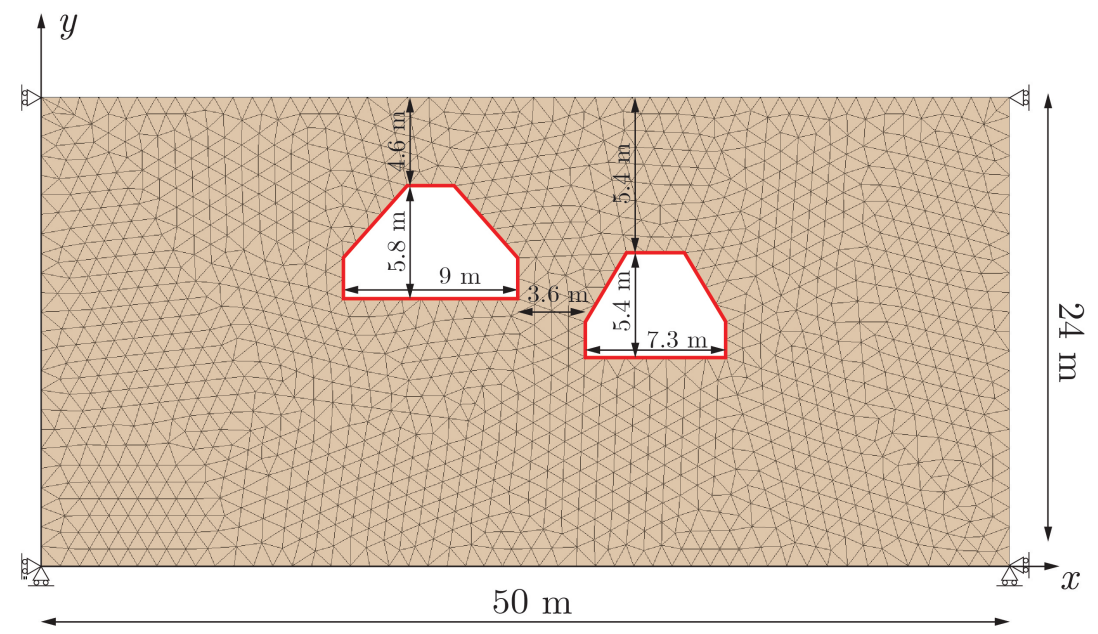

Fig. 16 Subsidence above underground cavities: problem geometry and FE discretization.

The geometry of the problem and the adopted FE discretization are shown in Fig. 16. Two parallel underground cavities of approximately trapezoidal shape in a calcarenite rock - similar to those typically found in underground quarries in the Puglia region, southern Italy - are exposed to a low $\mathrm{pH}$ environment, thus resulting in the development of a coupled chemo-mechanical diffusion and deformation process, triggered by rock degradation. In the simulations, the rock has been considered almost saturated due to capillarity effects (no STD and negligible suction effects), with pore water in hydrostatic equilibrium. The material constant set \# 1 has been adopted for the calcarenite rock.

Triangular T6P3C3 elements have been used in the FE discretization, which has approximately 11700 degrees of freedom. The elapsed time considered in the simulation is $t_{\text {fin }} \simeq 200$ days. Standard displacement boundary conditions have been applied to the external boundaries, with fixed horizontal and vertical displacements at the bottom and fixed horizontal displacements on the lateral boundaries. On the cavity surfaces, a fixed constant concentration of $\mathrm{H}^{+}$ions, corresponding to a $\mathrm{pH}$ of 4.5 , has been imposed.

The contour maps of debonding function $\mathcal{D}_{d}$ and of the internal variable $p_{t}$ at 3 different time stations, with $T=t / t_{\text {fin }}=0.23,0.46$ and 0.92 respectively, are shown in Fig. 17. As the diffusion process goes on, the progressive increase of $\mathcal{D}_{d}$ causes the reduction of rock stiffness and strength in a zone with almost constant tickness which spreads around each cavity with time. In the rock pillar between the two cavities, the two degradation zones merge together, enhancing the degradation effect.

Upon rock debonding, the self weight of the rock produces a displacement field which varies with time as shown in Figs. 18(a)-18(c), which provide the contour maps of the vertical displacement $u_{y}$ at $T=0.23,0.46$ and 0.92 . A close examination of Figs. 18(d)-18(f) - portraying the contour maps of the equivalent plastic strain $\epsilon_{\mathrm{eq}}^{p}$ at the same time stations - indicates that, at the beginning of the process $(T=0.23)$ the displacements are mostly caused by the reduction 


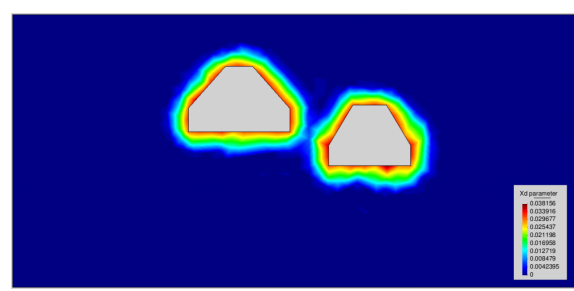

(a)

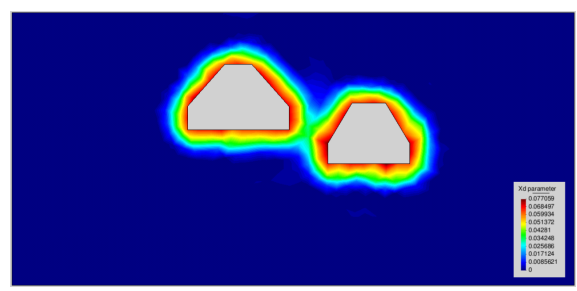

(b)

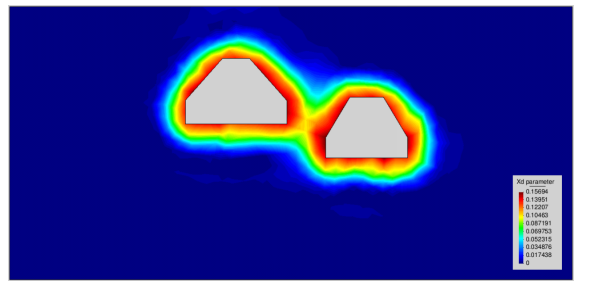

(c)

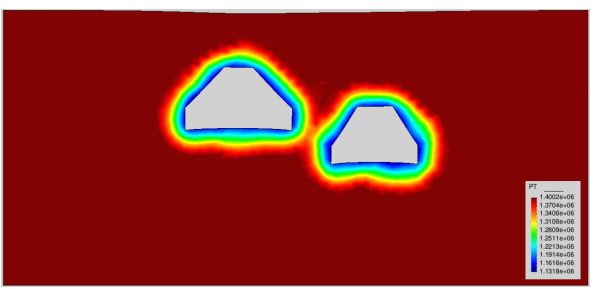

(d)

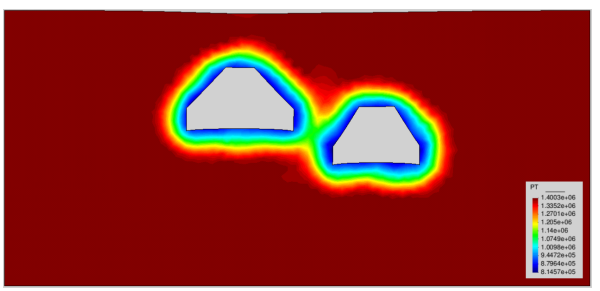

(e)

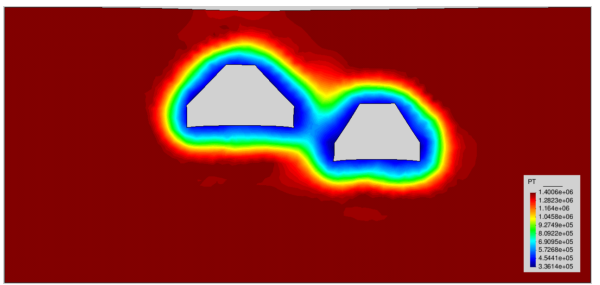

(f)

Fig. 17 Contour maps of: a) debonding function $\mathcal{D}_{d}$ at $T=0.23$; b) debonding function $\mathcal{D}_{d}$ at $T=0.46$; c) debonding function $\mathcal{D}_{d}$ at $T=0.92 ; \mathrm{d}$ ) internal variable $p_{t}$ at $T=0.23$; d) internal variable $p_{t}$ at $T=0.46 ; \mathrm{d}$ ) internal variable $p_{t}$ at $T=0.92$.

of the elastic stiffness of the material, whose stress state remains in the elastic range in most of the domain. As the degradation process continues, plastic zones starts forming on the sides of the central pillar, where compensation hardening takes place to maintain the consistency condition. At the same time, the stress redistribution which occurs in the pillar increases the elastic deformations of the still intact rock core. As a result, the vertical displacements increase more rapidly with time.

A more detailed picture of vertical displacements at ground surface is provided in Fig. 19. A well-defined settlement through forms and deepens with time, with a maximum settlement of about $11 \mathrm{~mm}$, located approximately above the crown of the shallower cavity.

The performance of the global Newton equilibrium iteration for 3 different time steps, chosen towards the end of the simulation when the degree of material non-linearity is highest, is shown in Fig. 20. As it can be observed, in all the steps convergence is attained with a very stringent relative tolerance (TOL = $1.0 \times 10^{-7}$ ) in no more than 9 iterations. All the profiles show an approximately quadratic convergence rate in their final part, demonstrating the effectiveness of the consistent linearization of the GBE algorithm presented in Sect. 5 . 


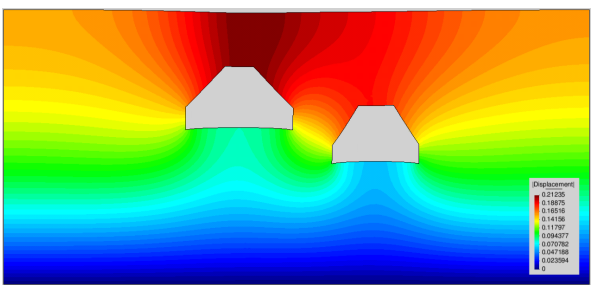

(a)

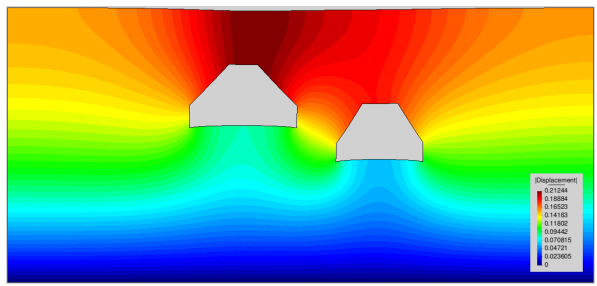

(b)

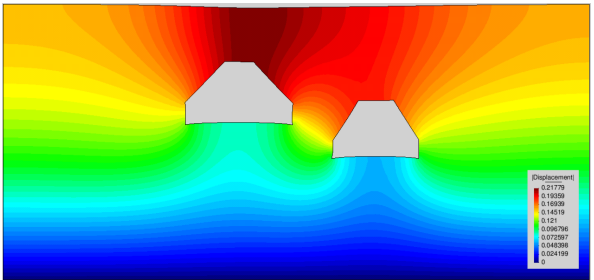

(c)

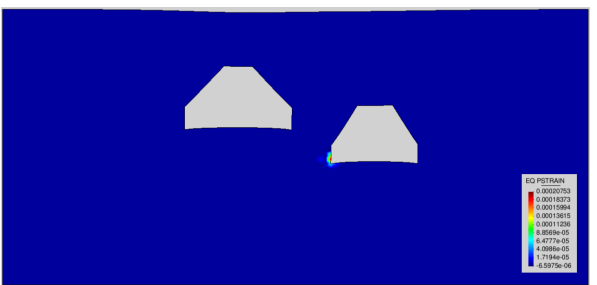

(d)

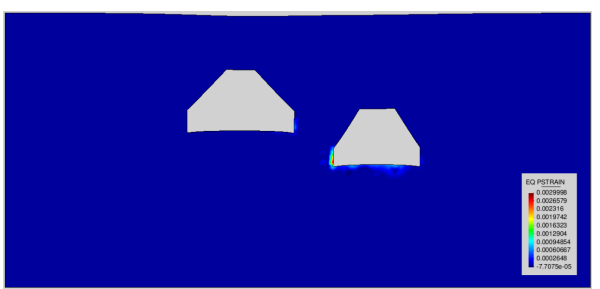

(e)

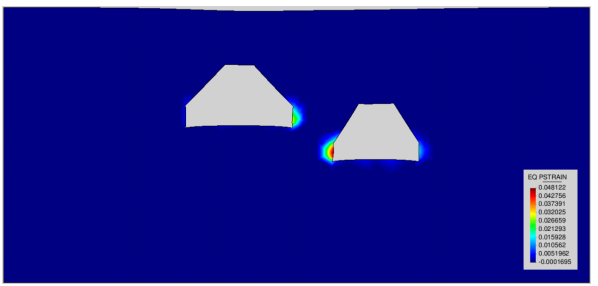

(f)

Fig. 18 Contour maps of: a) vertical displacement $u_{y}$ at $T=0.23$; b) vertical displacement $u_{y}$ at $T=0.46 ; \mathrm{c}$ ) vertical displacement $u_{y}$ at $T=0.92$; d) equivalent plastic strain $\epsilon_{\mathrm{eq}}^{p}$ at $T=0.23$; e) equivalent plastic strain $\epsilon_{\mathrm{eq}}^{p}$ at $T=0.46 ; \mathrm{f}$ ) equivalent plastic strain $\epsilon_{\mathrm{eq}}^{p}$ at $T=$ 0.92 .

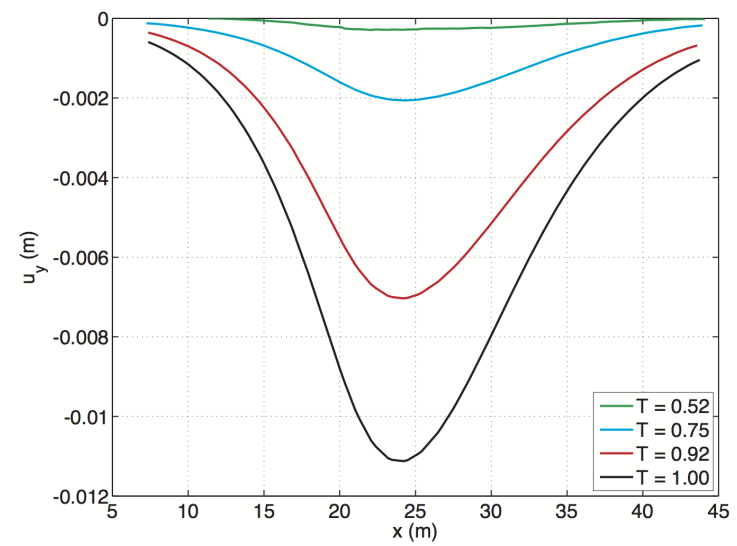

Fig. 19 Isochrones of ground surface vertical displacements for selected time stations. 


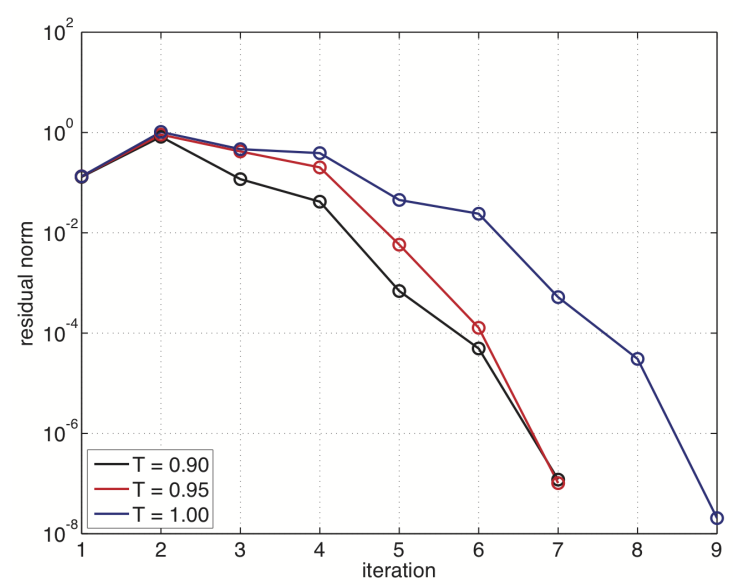

Fig. 20 Convergence profiles for selected time steps.

\section{Concluding remarks}

In this work, a general approach has been proposed to extend the classical theory of hardening plasticity to a wide range of natural geomaterials subject to "environmental" loading conditions, including wetting/drying processes, non-isothermal conditions and chemo-mechanical degradation.

In this approach, the coupling between the different physical processes is taken into account in two ways: by incorporating suitable relaxation terms in the expression of the constitutive stress rate, and by adopting generalized hardening laws for the internal variables, which allow them to vary as a consequence of both plastic deformations and of changes in the environmental process variables.

As an example, it has been shown how a recently proposed constitutive model for calcarenite rocks undergoing chemical debonding and dissolution [10] can be accomodated perfectly in the proposed theoretical framework.

A particular attention has been paid to the numerical issues related to the FE implementation of the proposed plasticity models with extended hardening, with a clear view towards their application to practical engineering problems. The additive structure of the generalized hardening law, coupled with the additive decomposition of strain rates, has allowed the development of a simple extension of the standard closest-point projection algorithms (GBE), widely used in computational mechanics for their excellent properties of stability and accuracy, to the proposed class of models.

The possibility of defining a suitable stored energy function for the elastic behavior of the material, and the simple structure of the phenomenological functions which quantify the environmental damage and degradation effects (for the particular case of the Ciantia and di Prisco model for calcarenites) has allowed to provide exact, closed-form solutions to the chemo-elastic predictor stage, even in presence of a strongly non-linear material response.

Remarkably, as all environmental degradation effects - including the development of chemical deformations - are dealt with in the predictor stage, the plastic corrector stage remains identical to that of classical elastoplasticity. Therefore, the 
proposed GBE algorithm preserves all the characteristics of stability and accuracy for large time steps typical of classical implicit return mapping schemes. Another important feature of the proposed algorithm is that it is amenable to closed-form linearization, thus allowing to exploit the quadratic convergence rate of Newton method in the solution of the discretized equilibrium equations.

The convergence and accuracy properties of the proposed GBE algorithm have been investigated by means of a large set of single-element test simulations. The results of these numerical experiments have demonstrated a good performance even for relatively large strain increments, which is not adversely affected by the incorporation in the model of such novel features as chemical deformations.

Finally, the simulation of two representative IBVPs - a chemo-hydro-mechanical OED compression test performed by [9] on a cylindrical specimen of Gravina calcarenite and the simulation of ground subsidence induced by rock degradation around underground cavities - has demonstrated the effectiveness of the proposed numerical integration strategy for the Ciantia and di Prisco model. The rapid convergence rate exhibited by some selected convergence profiles of the global equilibrium iterations in the underground cavities problem confirms the effectiveness of the proposed linearization procedure for the evaluation of the consistent tangent operators.

Acknowledgements The authors would like to warmly acknowledge the fundamental role played by their mentor and friend Roberto Nova in inspiring this work. The authors would like to thank Claudio di Prisco and Riccardo Castellanza for many fruitful discussions, and José Antonio Fernandez-Merodo for the support provided with the FE code GeHoMadrid. The financial support of the Project "PRIN 2010-11" is also gratefully acknowledged.

\section{References}

1. Alonso, E.E., Gens, A., Josa, A.: A constitutive model for partially saturated soils. Géotechnique 40(3), 405-430 (1990)

900 2. Bolzon, G., Schrefler, B., Zienkiewicz, O.: Elastoplastic soil constitutive laws generalized to partially saturated states. Géotechnique 46(2), 279-289 (1996)

3. Borja, R., Sama, K., Sanz, P.: On the numerical integration of three-invariant elastoplastic constitutive models. Comp. Meth. Appl. Mech. Engng. 192(9), 1227-1258 (2003)

4. Borja, R.I.: On the mechanical energy and effective stress in saturated and unsaturated porous continua. International Journal of Solids and Structures 43(6), 1764-1786 (2006)

5. Borja, R.I., Tamagnini, C.: Cam-clay plasticity, part III: Extension of the infinitesimal model to include finite strains. Comp. Meth. Appl. Mech. Engng. 155, 73-95 (1998)

6. Castellanza, R., Nova, R.: Oedometric tests on artificially weathered carbonatic soft rocks. Journal of geotechnical and geoenvironmental engineering 130(7), 728-739 (2004)

7. Cekerevac, C., Laloui, L.: Experimental study of thermal effects on the mechanical behaviour of a clay. International Journal for Numerical and Analytical Methods in Geomechanics 28(3), 209-228 (2004)

8. Ciantia, M., Castellanza, R., Crosta, G., Hueckel, T.: Effects of mineral suspension and dissolution on strength and compressibility of soft carbonate rocks. ENGGEO 184, 1-18 (2015)

9. Ciantia, M., Castellanza, R., di Prisco, C.: Experimental study on the water-induced weakening of calcarenites. Rock Mechanics and Rock Engineering 48(2), 441-461 (2014)

10. Ciantia, M., Prisco, C.: Extension of plasticity theory to debonding, grain dissolution, and chemical damage of calcarenites. International Journal for Numerical and Analytical Methods in Geomechanics (2015)

11. Ciantia, M.O.: Multiscale hydro-chemo-mechanical modelling of the weathering of calcareus rocks: an experimental, theoretical and numerical study. Ph.D. thesis, Politecnico di Milano (2013) 
12. Ciantia, M.O., Hueckel, T.: Weathering of stressed submerged calcarenites. Géotechnique 63, 768-785 (2013)

13. Ciantia, M.O., di Prisco, C., Castellanza, R.: Chemo-mechanical weathering of calcarenites: Experiments and theory. In: M. Manassero, A. Dominijanni, S. Foti, G. Musso (eds.) Coupled Phenomena in Environmental Geotechnics, pp. 541-548. Balkema, Rotterdam (2013)

14. Conti, R., Tamagnini, C., DeSimone, A.: Critical softening in cam-clay plasticity: adaptive viscous regularization, dilated time and numerical integration across stress-strain jump discontinuities. Computer Methods in Applied Mechanics and Engineering 258, 118-133 (2013)

15. Cui, Y., Delage, P.: Yielding and plastic behaviour of an unsaturated compacted silt. Géotechnique 46(2), 291-311 (1996)

16. Della Vecchia, G., Jommi, C., Romero, E.: A fully coupled elastic-plastic hydromechanical model for compacted soils accounting for clay activity. International Journal for Numerical and Analytical Methods in Geomechanics 37(5), 503-535 (2013)

17. van Eekelen, H.A.M.: Isotropic yield surfaces in three dimensions for use in soil mechanics. Int. J. Num. Anal. Meth. Geomech. 4, 89-101 (1980)

18. Fernandez-Merodo, J., Castellanza, R., Mabssout, M., Pastor, M., Nova, R., Parma, M.: Coupling transport of chemical species and damage of bonded geomaterials. Comp. \& Geotechnics 34(4), 200-215 (2007)

19. Fernandez Merodo, J.A., Mira, P., Pastor, M., Li, T.: GeHoMadrid User Manual. Internal Report, CEDEX, Madrid (1999)

20. Gens, A.: Soil-environment interactions in geotechnical engineering. Géotechnique 60(1), 3-74 (2010)

21. Gens, A., Guimaraes, L.d.N., Olivella, S.: Coupled chemomechanical analysis for saturated and unsaturated soils. Environmental geomechanics pp. 109-123 (2002)

22. Gens, A., Guimarães, L.d.N., Olivella, S.: Thmc coupling in partially saturated geomaterials. Revue européenne de génie civil 9(5-6), 747-765 (2005)

23. Hueckel, T.: Chemo-plasticity of clays subjected to stress and flow of a single contaminant. Int. J. Num. Anal. Meth. Geomech. 21, 43-72 (1997)

24. Jommi, C.: Remarks on the constitutive modelling of unsaturated soils. In: Experimental evidence and theoretical approaches in unsaturated soils, vol. 153. eds A. Tarantino and C. Mancuso. Rotterdam: Balkema (2000)

25. Jommi, C., Di Prisco, C.: A simple theoretical approach for modelling the mechanical behaviour of unsaturated soils. In: Proceedings of the Conference "Il ruolo dei fluidi nei problemi di Ingegneria geotecnica", vol. 1, pp. 167-188 (1994). (in Italian)

26. Lagioia, R., Puzrin, A.M., Potts, D.M.: A new versatile expression for yield and plastic potential surfaces. Comp. \& Geotechnics 19, 171-191 (1996)

27. Loret, B., Hueckel, T., Gajo, A.: Chemo-mechanical coupling in saturated porous media: elastic-plastic behaviour of homoionic expansive clays. International Journal of Solids and Structures 39(10), 2773-2806 (2002)

28. Maier, G.: On associative incremental elastic-plastic constitutive models. Rend. Ist. Lombardo di Scienze e Lettere 100, 809-838 (1966). (in Italian)

29. Nova, R.: Soil models as a basis for modelling the behaviour of geophysical materials. Acta Mechanica 64, 31-44 (1986)

30. Nova, R.: Modelling the weathering effects on the mechanical behaviour of granite. In: D. Kolymbas (ed.) Constitutive Modelling of Granular Materials. Springer, Berlin, Horton, Greece (2000)

31. Nova, R., Castellanza, R., Tamagnini, C.: A constitutive model for bonded geomaterials subject to mechanical and/or chemical degradation. International Journal for Numerical and Analytical Methods in Geomechanics 27(9), 705-732 (2003)

32. Nova, R., Castellanza, R., Tamagnini, C.: A constitutive model for mechanical and thermal loading of bonded geomaterials based on the concept of plasticity with extended hardening. Proc. NUMOG 9 (2004)

33. Ogden, R.: Nonlinear Elastic Deformations. Ellis Horwood, Chichester (1984)

34. Parise, M., Lollino, P.: A preliminary analysis of failure mechanisms in karst and man-made underground caves in southern italy. Geomorphology 134(1), 132-143 (2011)

35. Saetta, A., Scotta, R., Vitaliani, R.: Mechanical behavior of concrete under physicalchemical attacks. Journal of engineering mechanics 124(10), 1100-1109 (1998)

36. Saetta, A., Scotta, R., Vitaliani, R.: Coupled environmental-mechanical damage model of rc structures. Journal of engineering mechanics 125(8), 930-940 (1999) 
37. Schrefler, B.: The finite element method in soil consolidation (with applications to surface subsidence). Ph.D. thesis, University College of Swansea (1984)

38. Sheng, D., Sloan, S., Gens, A.: A constitutive model for unsaturated soils: thermomechanical and computational aspects. Computational Mechanics 33(6), 453-465 (2004)

39. Simo, J.: Numerical analysis and simulation of plasticity. Handbook of numerical analysis 6, 183-499 (1998)

40. Simo, J.C., Hughes, T.J.R.: Computational Inelasticity. Springer Verlag, New York (1997)

41. Simo, J.C., Taylor, R.L.: Consistent tangent operators for rate independent elastoplasticity. Comp. Meth. Appl. Mech. Engng. 48, 101-118 (1985)

42. Tamagnini, C., Castellanza, R., Nova, R.: A generalized backward euler algorithm for the numerical integration of an isotropic hardening elastoplastic model for mechanical and chemical degradation of bonded geomaterials. Int. J. Num. Anal. Meth. Geomech. 26, 963-1004 (2002)

43. Tamagnini, R.: An extended cam-clay model for unsaturated soils with hydraulic hysteresis. Géotechnique 54(3), 223-228 (2004)

44. Uchaipichat, A., Khalili, N.: Experimental investigation of thermo-hydro-mechanical behaviour of an unsaturated silt. Geotechnique 59(4), 339-353 (2009)

45. Wheeler, S., Sivakumar, V.: An elasto-plastic critical state framework for unsaturated soil. Géotechnique 45(1), 35-53 (1995) 